\title{
Mixing at Low Reynolds Numbers by Vibrating Cantilevered Ionic Polymers
}

\author{
Alicia Marie Williams \\ in partial fulfillment of the requirements for the degree of \\ Master of Science \\ In \\ Mechanical Engineering \\ Pavlos P. Vlachos, Committee Chair \\ Mark R. Paul, Committee Member \\ Mark A. Stremler Committee Member
}

Thesis submitted to the faculty of the Virginia Polytechnic Institute and State University

May 11, 2007

Blacksburg, Virginia

Keywords: Laminar Mixing, Nafion, Ionic Polymers, DPIV 


\begin{abstract}
Mixing at Low Reynolds Numbers by Vibrating Cantilevered Ionic Polymers
\end{abstract}

\title{
Alicia M. Williams
}

Creating mixing at low Reynolds numbers is a non-trivial challenge that has been approached from many different perspectives, using passive or active methods. This challenge been further highlighted with the rise of microfluidics. Based on the diminutive size of these devices, the Reynolds numbers are often less than 10, but have high Peclet numbers. Therefore, creating effective mixing is non-trivial and is a topic of active research, and is of paramount importance in order to improve performance of microfluidic devices in a wide range of applications.

The objective of this research was to develop a novel active device for laminar mixing. The mixing device developed herein capitalized on Nafion ionic polymers, which are a class of active materials that are thin, flexible, inexpensive, and readily deployable in an aqueous medium and offer strains up to $5 \%$ under a small $(<2 \mathrm{~V})$ applied voltage. The effect of these deflections on an incident flow is the mixing mechanism in a laminar channel flow explored in this effort.

To the author's knowledge, the high-risk effort presented herein is the first attempt to exploit ionic polymers as an active mixing device. Several different configurations of ionic polymers were tested and Digital Particle Image Velocimetry (DPIV) measurements were obtained. Resulting analysis using a quantitative mixing metric shows that using cantilevered polymers create increases mixing potential in the flow for some actuation cases. Although these differences are present, they do not appear consistently in the results. However, only a partial set of flow information was obtained from DPIV, and an improved understanding of the effect of these polymers could be developed from additional experiments.

Using cantilevered ionic polymers for laminar mixing could foster the development of a new generation of efficient micromixing devices, which will improve the capabilities and effectiveness of numerous microfluidic technologies that range across biomedical, lab-on-a-chip, separation and sorting technologies and many more. 


\section{Acknowledgements}

I'd like to begin first by recognizing the National Science Foundation Graduate Research Fellowship for providing funding for me to conduct the research that is the subject of this thesis. I would also like to acknowledge all the members of my committee for their advice and suggestions, as well as their patience while I finished up the final parts of the thesis. To my advisor, Pavlos, I am deeply grateful for how he has served as both my mentor and friend for the past few years. For me, graduate school has turned into a much different experience than I envisioned before I began, in that I've learned more than I could have imagined, and I owe that to Pavlos.

A huge thanks goes to all the great people with whom I have had the privilege to work with over the past two years in the lab. I will take this opportunity also to recognize my late friend and colleague Patrick Leung, who was an inspiration to all of us in that he was such a giving, honest, and hardworking individual. I honestly feel that I could not have better people to share the graduate school experience with, and I am honored to know all of you. I'd also like to recognize the often overlooked ME staff, who have always been a great help to me in so many ways, especially Cathy Hill, Peggy Caldwell, and Lisa Stables.

Lastly, but absolutely not least, is my family and Amanda. I can't even begin to say how amazing it has been to see how much my family has supported and helped me out, especially during the past few months during the preparations for my defense. But beyond that, I have come to appreciate how special it is that my parents always encouraged me to follow my dreams and never give up, even when things don't go as expected. Big thanks also for being patient during the phone calls during which I would explain the latest difficulty with my experiments. Finally, Amanda is someone without whom I would not be finishing my degree. I cannot express how happy I am to have you in my life, and I am so grateful to you for all your support and willingness to put up with me, especially when I was nearing completion of this work. 
To my parents, who have always encouraged me to follow my dreams 


\section{Contents}

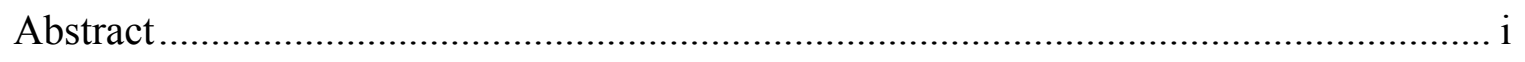

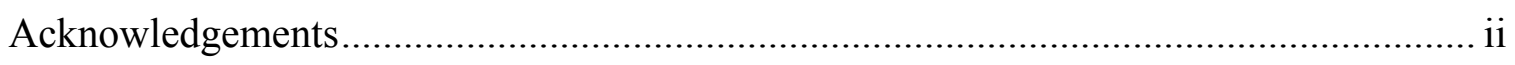

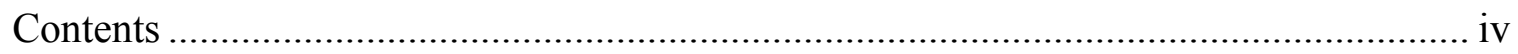

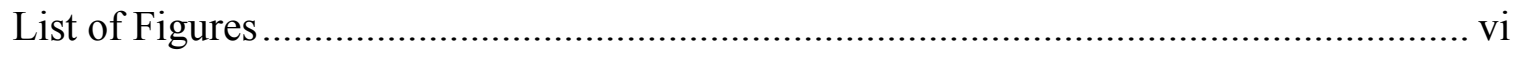

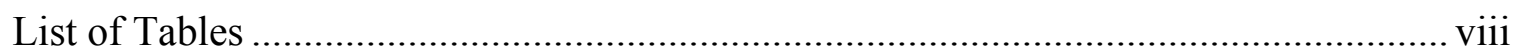

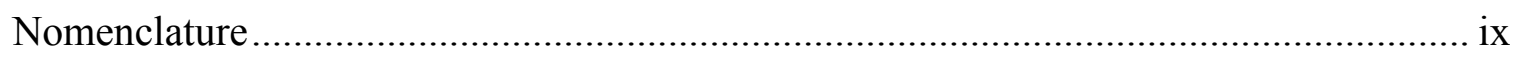

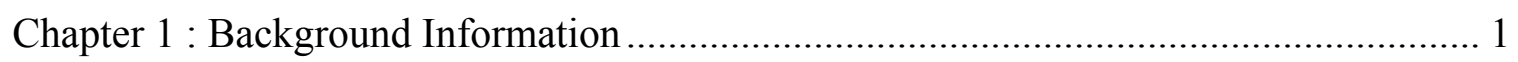

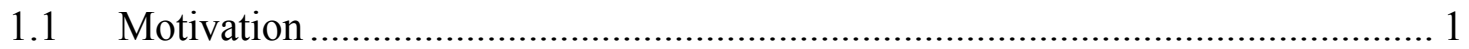

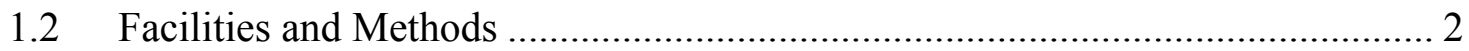

1.3 Our Previous Efforts Using Ionic Polymers for Active Mixing............................ 3

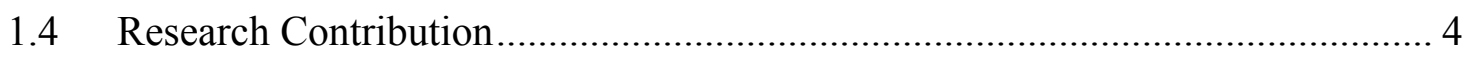

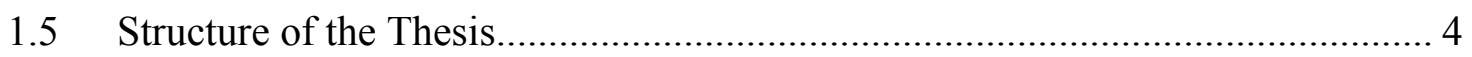

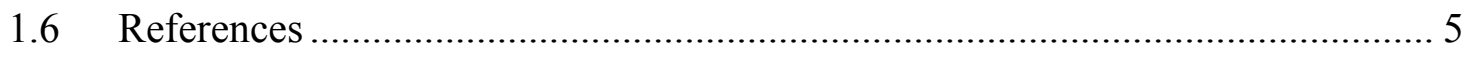

Chapter 2 : Study of Oscillating Cantilevered Polymer in Low Reynolds Number Flow.. 6

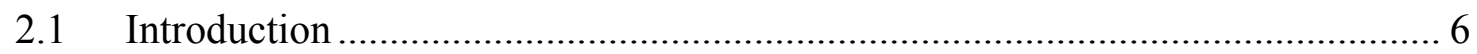

2.2 Experimental and Analysis Tools and Methodology ………………................. 9

2.2.1 Nafion Ionic Polymer Construction and Operation ..................................... 9

2.2.2 Experimental Facilities and Setup ........................................................ 12

2.2.3 Experimental Setup ..................................................................... 15

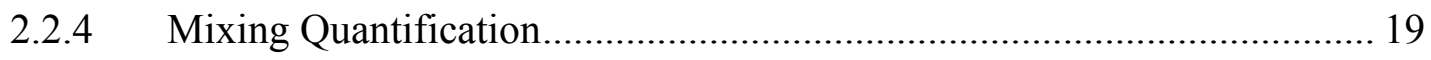

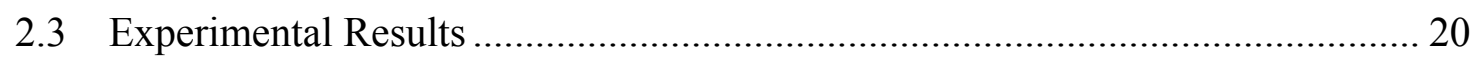

2.3.1 DPIV Post Processing and Data Nomenclature ........................................... 21

2.3.2 Results for Selected Polymer Configurations .............................................. 21

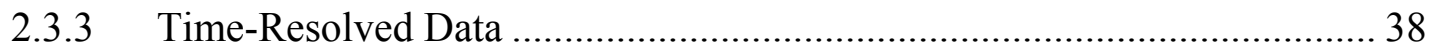


2.4 Discussion and Summary of Results............................................................. 40

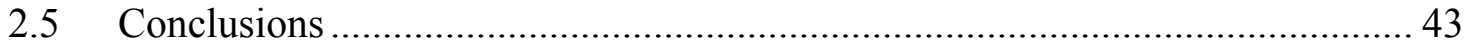

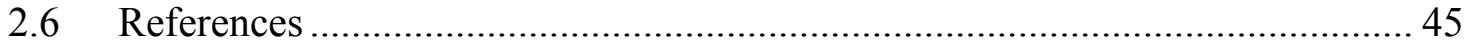

Chapter 3: Conclusions and Recommendations ............................................................ 47

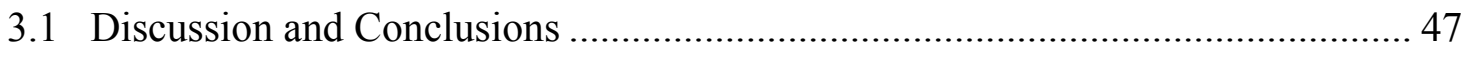

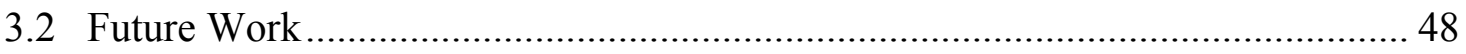

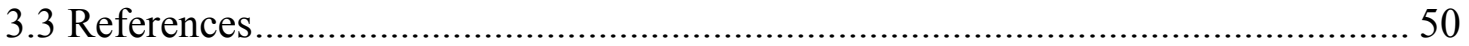

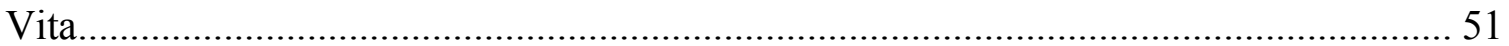




\section{List of Figures}

Figure 1.1. A typical DPIV experimental set up, with a laser plane illuminating flow tracer particles.

Figure 2.1 Schematic showing ionic polymer construction and deflection.

Figure 2.2. Process for creating an ionic polymer actuator.

Figure 2.3. Channel used for mixing polymer testing. The top of the channel (shown in black) is removable to allow for placement of multiple polymer configurations.

Figure 2.4. Schematic showing how ionic polymers deflect with respect to changes in voltage polarity.

Figure 2.5. DPIV configuration for orthogonal ROI testing to obtain flow structures in the cross section of the channel.

Figure 2.6. DPIV configuration for streamwise flow data acquisition. The laser plane was located at the channel centerline.

Figure 2.7. Mixing polymer configurations. A) Shows a four polymer configuration with polymers in the "parallel" orientation. B) Shows a four polymer configuration with polymers in the "orthogonal" orientation.

Figure 2.8. Schematic showing the relative location of the parallel-oriented single polymer with respect to the laser plane.

Figure 2.9. Normalized velocity magnitudes for baseline and actuation cases of 1 parallel polymer at its trailing edge.

Figure 2.10. Percent difference in velocity magnitude for 1 parallel polymer at $0 \mathrm{~mm}$ downstream

Figure 2.11. Mixing potential, $\xi$, for 1 parallel-oriented polymer at $0 \mathrm{~mm}$ downstream. 25

Figure 2.12. Difference in mixing potential, $\xi$, for one parallel-oriented polymer at its trailing edge.

Figure 2.13. Schematic illustrating relative orientation of the polymer with respect to the laser plane

Figure 2.14. Time-averaged baseline and actuation velocity fields normalized by the mean streamwise velocity for one orthogonally-oriented polymer at its trailing edge.....27 
Figure 2.15. One orthogonally-oriented polymer, showing the percent difference in velocity magnitude between baseline and actuation.

Figure 2.16. Mixing potential, Xi, for one polymer for baseline and actuation with a measurement plane located at the trailing edge of the polymer...... 30

Figure 2.17. Percent difference in the mixing potential, $\xi$, for one orthogonally-oriented polymer at its trailing edge.

Figure 2.18. Percent difference in velocity magnitude for 1 orthogonally-oriented polymer at 5 and $10 \mathrm{~mm}$ downstream.

Figure 2.19. Normalized velocity magnitudes for four orthogonally-oriented polymers, 5 mm downstream.

Figure 2.20. Normalized velocity magnitude for four orthogonal polymers, $10 \mathrm{~mm}$ downstream.

Figure 2.21. Percent difference in velocity magnitude between baseline and actuation cases for 4 orthogonally-oriented polymers at locations of A) 5 millimeters and B) 10 millimeters downstream.

Figure 2.22. Mixing potential of 4 orthogonal polymers at $5 \mathrm{~mm}$ downstream.

Figure 2.23. Mixing potential for a 4 orthogonal polymer configuration at $10 \mathrm{~mm}$ downstream.

Figure 2.24. Percent difference in mixing potential, $\xi$, for four orthogonal polymers at A) $5 \mathrm{~mm}$ downstream, and B) $10 \mathrm{~mm}$ downstream.

Figure 2.25. Time series of $\xi$ for a 4 orthogonally-oriented polymer case averaged over the field of view at a location $10 \mathrm{~mm}$ downstream of the last polymer in the configuration.

Figure 2.26. Comparison of time-resolved $\xi$ averaged over each instantaneous field of view for a four orthogonal polymer configuration

Figure 2.27. Comparison of percent difference of mixing for all configurations tested. 41

Figure 2.28. Percent difference in $\xi$ based on a clean channel as a baseline, compared to polymer actuation 


\section{List of Tables}

Table 2.1 A summary of relevant dimensionless parameters. .................................... 13

Table 2.2. Test matrix for a single ensemble of polymer testing. An additional ensemble of data was acquired one day after this data set...................................................... 18 


\section{Nomenclature}

$\begin{array}{ll}A_{T} & \text { Amplitude of cantilevered polymer tip deflection } \\ D_{h} & \text { Hydraulic diameter } \\ D_{i f f} & \text { Molecular diffusivity of water, } 0.2272 * 10^{9} \mathrm{~m}^{2} / \mathrm{s} \\ e_{i j} & \text { Rate of strain tensor (two-dimensional) } \\ l_{e} & \text { Entrance length needed for fully developed flow } \\ P_{D i f f} & \text { Percent difference between normalized baseline and actuation velocities } \\ P e & \text { Peclet number } \\ R e & \text { Reynolds number } \\ U_{T} & \text { Tip velocity of an actuating cantilevered polymer } \\ v & \text { Kinematic viscosity (water for all calculations) } \\ v & \text { Component of velocity in the flow cross section in the y-direction } \\ V_{a c t u a t i o n} & \text { Velocity (i,j) measured during polymer actuation } \\ V_{b a s e l i n e} & \text { Velocity (i,j) measured with the polymer at baseline } \\ w & \text { Component of velocity in the flow cross section in the z-direction } \\ z / D_{h} & \text { Normalized z-coordinate } \\ y / D_{h} & \text { Normalized y-coordinate } \\ \xi & \text { Mixing potential (magnitude of the rate of strain tensor) }\end{array}$




\section{Chapter 1 : Background Information}

\subsection{Motivation}

Microfluidics is a rapidly growing research field [1] with a substantial range of applications, some examples of which include: DNA and biological analysis [2], detection of chemicals or pathogens [3], and in vivo biological monitors [4]. Moreover, as a part of the economy, microfluidics continue to gain momentum. This is particularly true for those with biological applications, with a potential market of billions of dollars $[5]$.

Many microfluidic applications require mixing to foster chemical reactions, which for macroscale flows is typically achieved with turbulent flow regimes. Although turbulent flow is often regarded as undesirable in many different systems, its chaotic nature is advantageous in devices that require increased mixing such as internal cooling passages of turbine blades for improvement of heat transfer [6]. However, in contrast to traditional mixing problems, microscale flows generally have Reynolds numbers on the order of 100 or lower, implying that the flow is predominantly laminar and governed by viscous effects. As a result, high levels of mixing do not occur naturally. Also, these flows tend to have a high Peclet number, which is a ratio of the advection of the fluid to its diffusion. For microscale flows, the Peclet number is typically greater than 100, indicating that the timescale of material convection downstream of the channels is faster than the timescale of molecular diffusion.

Based on the extremely limited ability for efficient mixing to occur in a flow with such Reynolds and Peclet numbers, specialized devices are needed to improve the performance of microfluidics so that they can compare and compete with macroscale devices. Specifically, in order to be useful for rapidly developing microfluidics technology, these mixing devices must be inexpensive, small (including packaging), 
immersible in aqueous environments, simple to manufacture, and of course efficient in mixing.

This work demonstrates the development of a novel device that promises to fulfill all of these needs using actively driven Nafion ${ }^{1}$-based cantilevered ionic polymer actuators to enhance mixing. Ionic polymers offer great potential as actuators for such applications due to the low voltage supplies required, usability in liquid environments, their ability to generate very large strains and the fact that they can easily and inexpensively be fabricated in almost any size and shape.

\subsection{Facilities and Methods}

The flow structures and velocities created by the polymer motion are of interest to characterize the performance of the device. To measure these quantities in the field of view that encompasses the flow cross-section, Digital Particle Image Velocimetry (DPIV) [7] is the most readily efficient and established method to obtain a large number of point velocity measurements simultaneously. DPIV is a non-invasive optical flow measurement method that combines a pulsing laser synchronized with a high speed digital camera to obtain planes of velocity vectors, with potential sampling frequencies in excess of $1 \mathrm{kHz}$ if time resolved data are desired.

However, low sampling frequency data acquisition was more than sufficient due to the low Reynolds numbers encountered in this work ( $\operatorname{Re} 10$ for all polymer configurations examined). The pulsing laser used in DPIV experiments is opened to a plane of 1-2 mm thickness using a cylindrical lens in the region of interest and illuminates flow tracer particles. The particles are neutrally-buoyant and follow the flow without time delay. A Complementary Metal Oxide Semiconductor (CMOS) camera images the particles in the laser plane, and is synchronized with the laser pulses using a timing board. The displacement of the particles between successive frames and the pulse separation of the laser beam are used in concert with cross-correlation algorithms to

\footnotetext{
${ }^{1}$ Nafion ${ }^{\circledR}$ is a sulfonated tetrafluorethylene copolymer discovered in the late 1960 s by Walther Grot of DuPont. It is the first of a class of synthetic polymers with ionic properties which are called ionomers.
} 
calculate velocity vectors. An illustration of a generalized DPIV setup used for data acquisition is shown in Figure 1.1.

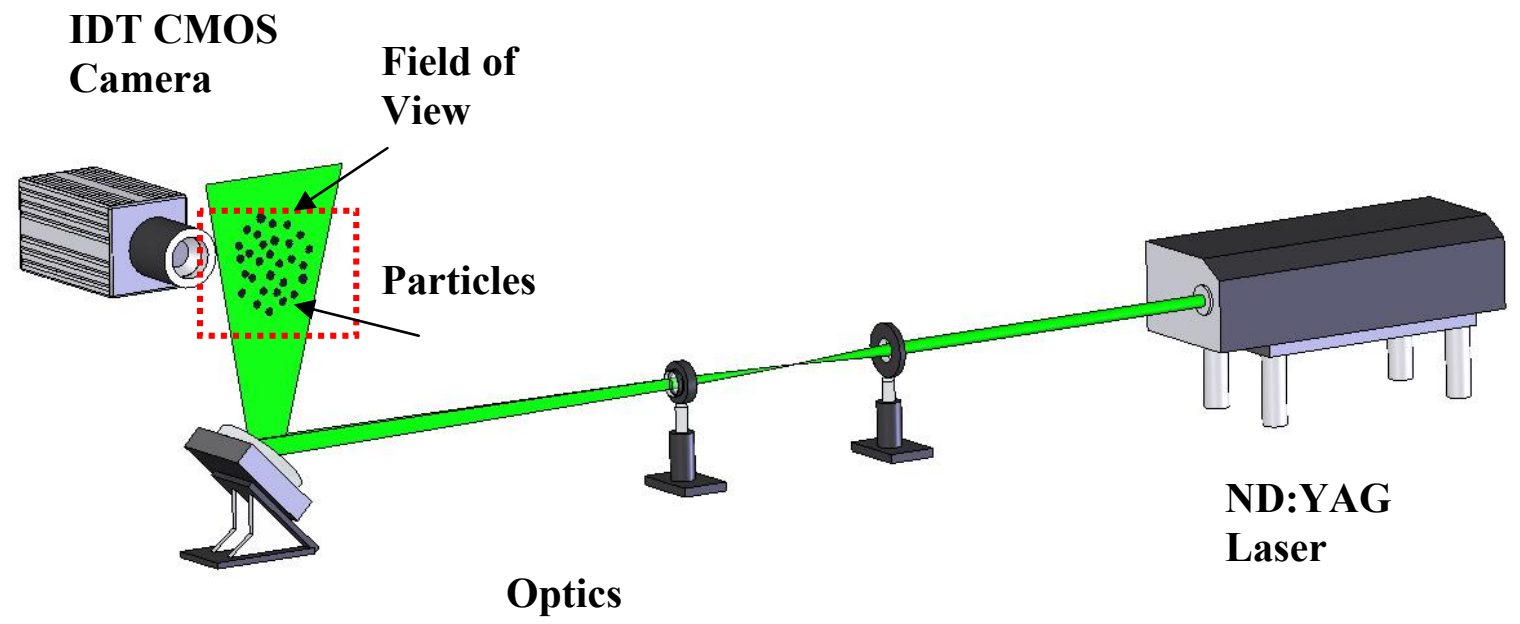

Figure 1.1. A typical DPIV experimental set up with a laser plane illuminating flow tracer particles.

A channel was designed to contain the polymer cantilevers and provide optical access for DPIV data. Additional information about the test section and experimental facilities will be presented in Chapter 2.

\subsection{Our Previous Efforts Using lonic Polymers for Active Mixing}

A first experiment was conducted in which these polymers were placed in a stagnant flow to determine how they could induce flow structures due to their vibration. A key difference between this first experiment and the work presented in this thesis was that a different actuation configuration was implemented in the form of an active wall/skin membrane and in that the experiment was performed in quiescent flow conditions. The polymer deflections were on the order of 5 microns. This experiment 
also used DPIV as the flow diagnostic technique. It was later determined that the flow was dominated by natural convection effects due to the heating from the laser, under stagnant flow conditions. To circumvent the issue of heating from a laser, the experiment that is the subject of this thesis made use of flow in a low Reynolds number channel. In this fashion, fluid elements that enter the laser plane exit before significant heating can occur. Using a basic analysis with the First Law of Thermodynamics, it was estimated that a maximum of 2 degrees Fahrenheit could occur before the fluid exits the laser plane, even if all the laser energy was absorbed.

\subsection{Research Contribution}

The goal of this work was to utilize cantilevered Nafion ionic polymer membranes in a cantilevered configuration to develop an efficient low Reynolds number mixing or stirring device. The distinction between mixing and stirring is an important one, and was first described by Reynolds in 1894 [8]. Stirring denotes the distribution of a material throughout a medium, but mixing refers to the final diffusion of material across the interfaces to create a completely homogeneous species. The analysis presented in this work does not take diffusion into account and focuses on the improvement in stirring due to the use of these polymers in actuation mode. Therefore where mixing is stated within this text, mixing due to only stirring is implied. To the author's knowledge, this is the first usage of these active polymer transducers to increase the mixing in a flow. To evaluate the polymer's mixing capabilities, DPIV measurements were obtained with two different experimental setups using an $\mathrm{Nd}$ :Yag laser viewing both the centerline streamwise planes of the channel as well as the cross-sectional plane of the channel at several locations at and downstream of the polymer configurations.

\subsection{Structure of the Thesis}

First, the motivation behind this work as well as a current state of the art in laminar mixing devices will be presented. Second, the experimental setup and analysis 
methodology will be discussed, and the results of the experiment will be presented. This thesis will present the data obtained predominantly from the cross-sectional DPIV plane, as this is the plane in which we anticipated to measure the largest impact. An analysis of the impact of the different configurations of the polymer on the overall flow will also be presented. Finally, the conclusions of this experiment along with recommendations for future work will be discussed.

\subsection{References}

1. Stone, H.A. and S. Kim, Microfluidics: Basic issues, applications, and challenges. Aiche Journal, 2001. 47(6): p. 1250-1254.

2. Yi, C., Li, C.-W., and Yang, M: Microfluidics technology for manipulation and analysis of biological cells. Analytica Chimica Acta, 2006. 560 (1-2): p. $1-23$.

3. Jakeway, S.C., de Mello, A.J., and Russell, E.L., Miniaturized total analysis systems for biological analysis. Fresenius Journal of Analytical Chemistry, 2000. 366 (6-7): p. 525-539.

4. Phayre, A. N., N. K. Hartley, and M. A. Hayes. Microdevices for biological analyses recent advances and directions for the future. Journal of the Association for Laboratory Automation, 2000. 5 (4): p. 78-82.

5. Jain, K.K., Biochips and Microarrays:Technology and Commercial Potential. Pharmaceutical Industry Report, 2000.

6. Iacovides, H. and M. Raisee, Recent progress in the computation of flow and heat transfer in internal cooling passages of turbine blades. International Journal of Heat and Fluid Flow, 1999. 20(3): p. 320-328.

7. Westerweel, J., Fundamentals of digital particle image velocimetry. Measurement Science \& Technology, 1997. 8(12): p. 1379-1392.

8. Reynolds, O., Study of fluid motion by means of colored bands. Nature, 1894. 50 p. 161-164. 


\section{Chapter 2 : Study of Oscillating Cantilevered Polymer in Low Reynolds Number Flow}

\subsection{Introduction}

Microchannels have become increasingly prevalent within MEMS and NEMS research in a wide range of applications, including: biological analysis [1], small volume transport and analysis [2], and bioengineering [3]. Moreover, using microscale systems for these applications and beyond represents a more efficient (smaller quantities of liquid can be used for analysis) and cost effective alternative [4]. Based on the wide variety of applications coupled with their overall promise, microfluidics are expected to have an enormous impact on the economy [5].

Mixing is often necessary in microfluidic applications, for example, to speed chemical reactions and perform rapid analysis. However, due to the low Reynolds numbers that characterize small and microscale flows, it is non-trivial to generate interactions of the fluid with itself, unlike at larger scales. The primary mechanism that fosters mixing at low Reynolds numbers is diffusion, which requires prohibitively long time (or channel length) scales to be effective. The wide range of mechanisms that have been developed to improve mixing at small scales of flows are as varied as the applications for microfluidics. However, most can be grouped primarily by those that act passively on the flow, and active devices that require energy input to create mixing.

Many passive mixing devices presented to date vary the geometry of the channel itself to create interacting twisting flow structures, such as the serpentine microchannel studied by Liu et al [6]. This microchannel is comprised of repeating C-shaped sections which show increased mixing ability compared to straight channels as a result of the twists and turns that the flow experiences. For a Reynolds number of 70, the authors demonstrated 16 times more reacted phenolphthalein corresponding to the interaction of 
two originally separated streams within the serpentine channel compared to a straight channel. In addition to altering the flow path by use of complex channel geometry, a readily created mixing device involves the insertion of obstructions into the flow cavity. Riblet-lined channels have been studied at length as a passive means to improve heat transfer by enhancing turbulence $[7,8,9]$, and this technology has also been examined at the smaller scales. One example of a ribbed or grooved passage is a microchannel with rectangular-shaped grooves in a staggered herringbone pattern [10]. This channel creates counter-rotating vortices in the flow, and by varying the herringbone's shape, an interaction of these vortices is created. These structures resulted in an increase of 90 percent more mixing relative to a smooth channel over a 0.5 centimeter length, which is 65 channel heights.

In contrast to the passive mixers, active mixing devices such as pulsed micropumps can create mixing over shorter lengths than passive devices, but require an active input of some form of energy. Aubry's group [11] has shown that pulsing a micropump in microscale channels with a T-junction inlet can be effective at mixing two fluids. Also, a recent paper by the same group has shown that pulsed mixing combined with geometrical variations in the channel geometry can hasten the mixing at microscales [12]. Other studies have used microfabricated pumps themselves to overcome diffusion and mix species within the pump geometry, such as that by Chou, et al [13]. This rotary pump, constructed using soft lithography, can peristaltically pump fluid around a loop which comprises the pump geometry. The peristalsis rate of the pump was controllable, and the device has several ports to allow for mixing of a specific volume of fluid or for mixing a continuous flow. Analytical models of peristaltic pumps based on sinusoidal boundary displacements have been described by Selverov [14], and Yi [15]. Yang et al [16] developed a valveless micropump that mixes using ultrasonically vibrating piezoceramic actuators.

Another set of active mixing devices uses applied external fields of a variety of types as the mechanism to act on the flow and create mixing. The majority of these devices presented to date utilize either electrical or magnetic fields. Aubry's group has developed an Electrohydrodynamic (EHD) mixer [17], which operates on the premise of 
mixing two fluid species that have different electrical properties. The EHD mixer was tested for both AC and DC applied electrical potentials of 100 to 300 Volts and achieved excellent results, mixing the flow in less than 250 microns of channel length for a Reynolds number of 0.02. Another example is found using a two-well electroosmotic mixer, where each well was supplied with a non-zero offset and a time-varying square wave voltage [18]. Modifying the time-varying component of voltage created a pulsating flow which ultimately mixed the fluids from the two wells. A similar device based on applied magnetic fields was developed by applying a current to a conductive fluid within the microchannel to create a Lorentz body force [19]. By varying the Lorentz forces in time, fluid stretching occurred; this increased the interfacial area and hence mixing between a water-soluble dye and water.

Many active mixing devices are sophisticated based on the demands of laminar mixing, and some have complex moving parts. This work seeks to develop an active mixing device not dependent on generation of external fields or on mechanical parts. The mechanism used to create flow structures is a vibrating cantilevered polymer membrane using an active material in the form of Nafion ionic polymer transducer. Previous work studying the flow generated by small vibrating cantilevers created with piezoceramics has been performed by Kim, Wereley, and Chun [20]. In their work, vortex shedding was captured off the tip of the vibrating piezeoceramic cantilever in quiescent air with DPIV. The Reynolds numbers in this study spanned 40 to 150 based on the cantilever vibration of $180 \mathrm{~Hz}$ and the amplitude of vibration at its tip. Piezoceramics, however, are not as practical for small scale application as they produce less strain than ionic polymers, and generally require 100 or more Watts of power to create any sizeable deflection compared to 0.4 Watts for ionic polymers. Additional information about ionic polymers will be presented in the proceeding section.

The objective of this work was to examine, for the first time, the effect of these actively vibrating ionic polymer cantilevers on a laminar flow with a Reynolds number of 10 based on the average streamwise velocity of the channel flow. The polymer was driven at 1 Hertz with a 1.5 Volt amplitude square wave. Alternating the voltage polarity of the polymer cantilevers is necessary to produce a constant vibrating motion at the tip 
of the polymer. The results presented were quantified using DPIV (Digital Particle Image Velocimetry) sampling at $128 \mathrm{~Hz}$.

\subsection{Experimental and Analysis Tools and Methodology}

\subsubsection{Nafion Ionic Polymer Construction and Operation}

The material used for this active mixing device was ionomeric electroactive polymers, which are a class of active materials which react to changes in their environment by either producing a strain under applied voltage or a charge when under stress. The ionic polymer cantilevers consisted of an ion-exchange membrane sandwiched between two layers of conductive metal (gold and platinum) as shown in Figure 2.1. For this work, the ion-exchange membrane used to create these polymers is Nafion, a material product of DuPont, and the membrane was saturated with water to provide a medium for ion transport. The Nafion membrane was 180 microns thick, with electrodes on either side of 20 micron thickness.

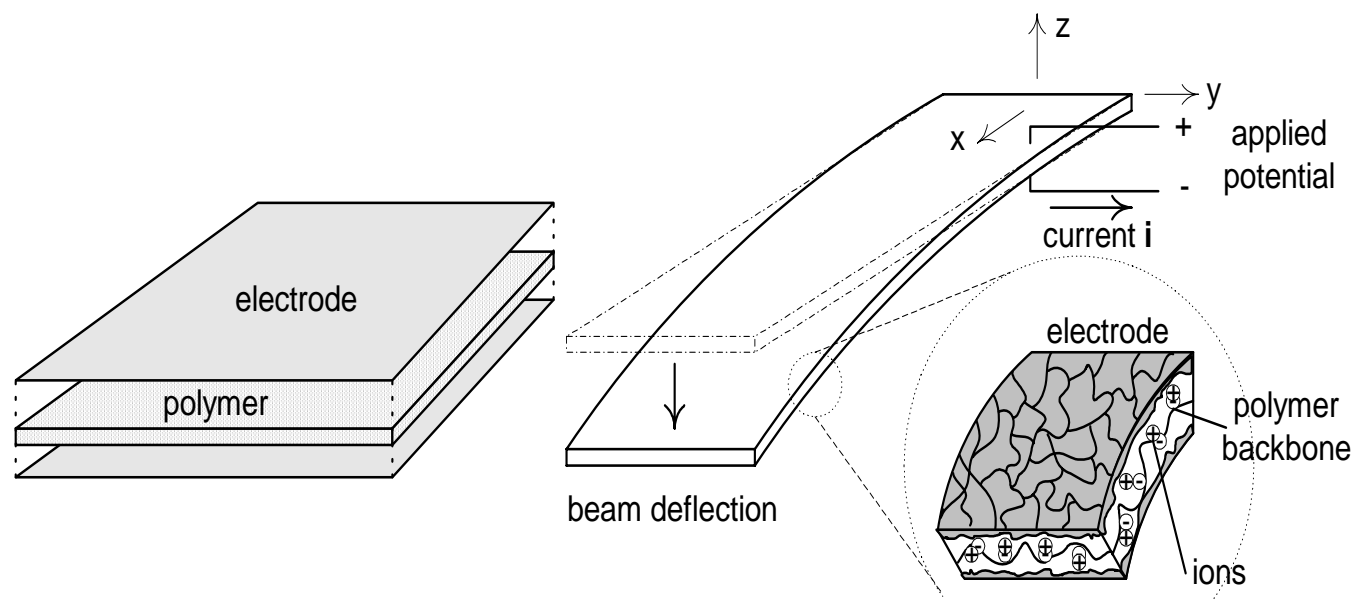

Figure 2.1 Schematic showing ionic polymer construction and deflection. 
Ionic polymers exhibit electromechanical coupling that allows them to be used as either sensors or actuators, based on whether a voltage or a force is applied to the polymer. The ionic polymers were utilized in actuation mode, wherein a small voltage $(<2 \mathrm{~V})$ is applied across the polymer electrodes to create the desired deflection. The free cations within the Nafion membrane become attracted to the anode and gather near the electrode surface, drawing the liquid within the polymer with it, which was de-ionized water for this polymer type. The migration of ions and water on one side of the polymer creates a stress due to the additional pressure of the water, and this is the mechanism responsible for the deformation of the polymer. The response of the polymer varies nonlinearly as a function of the voltage and the inverse of frequency. The deflections with respect to both voltage and frequency follow a non-linear decaying curve, where the highest deflections are obtained for the highest applicable voltage and low driving frequency, near $1 \mathrm{~Hz}$.

A comparison between ionic polymers and piezoceramics may be drawn, as piezoceramics similarly produce deflections under applied voltage potentials. However, piezoceramics require a much higher driving voltage (on the order of 100 or more volts) to produce any significant deflections. In spite of this higher applied voltage to the piezoceramic, only $0.1 \%$ strain is possible compared to $5 \%$ strain possible for ionic polymers [21]. Another advantage of ionic polymers is that their final geometries are readily alterable as they are constructed of thin (200 micron thickness or less) and flexible membranes.

The particular ionic polymer type investigated for this research was developed using a novel fabrication technique by Akle et al. [22], which enables large strains while incorporating inexpensive electrode materials such as $\mathrm{RuO}_{2}$ and Carbon black. The construction process for these particular polymers is shown in Figure 2.2. First, the electrode mixture is prepared, and is comprised of $\mathrm{RuO}_{2}, 5 \%$ liquid Nafion solution, and de-ionized water. The Nafion membrane is separately boiled first in $\mathrm{H}_{2} \mathrm{SO}_{4}$, then $\mathrm{LiOH}$. A final boiling in de-ionized water cleans the Nafion of debris from the first two solutions. The lithium that resides on the membrane at the end of the boiling process in $\mathrm{LiOH}$ acts as the free cation for ion exchange. The completed electrode mixture is 
manually sprayed onto the entire surface of the Nafion membrane after the final boiling. Then, the polymer composite of the electrode material that sandwiches the Nafion membrane is melt pressed at high temperature and pressure to ensure a solid connection between the Nafion and the electrodes. Once the composite is melt pressed, an impregnation reduction process is used to embed platinum onto and inside the ruthenium dioxide electrode. The $\mathrm{RuO}_{2}$ does not have excellent conductivity, so the purpose of the impregnation reduction is to create small dendritic structures that penetrate the electrode and can conduct directly to the Nafion membrane. The final step of the manufacturing process is to use electrolysis to plate the entire electrode with gold, as to increase the surface conductivity of the electrodes.

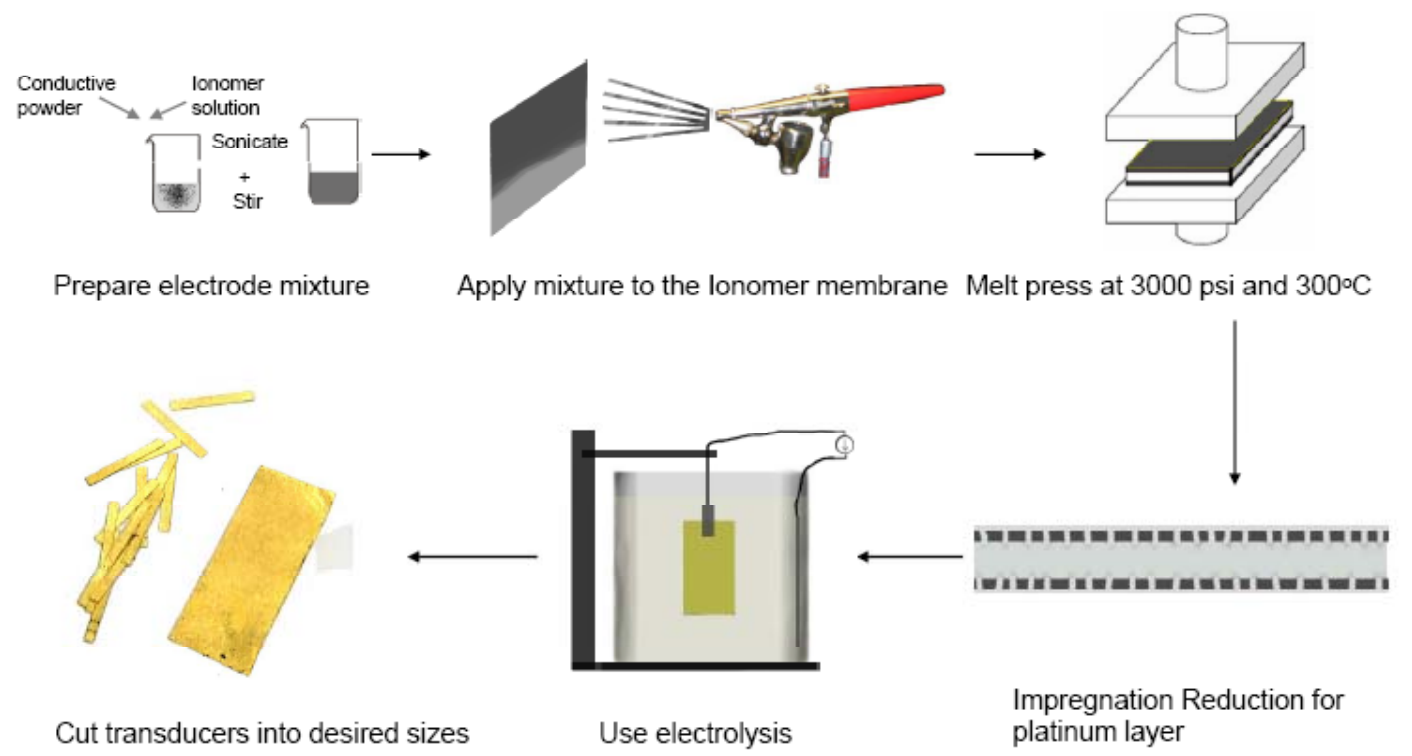

Figure 2.2. Process for creating an ionic polymer actuator.

These polymers are water-based, so they are readily immersible in aqueous environments with no additional modifications. One drawback to these polymers is their dehydration (and subsequent decreased response to applied voltages) over time when actuated in a gaseous environment, but this issue does not apply to this work, as it was conducted entirely in liquid media. However, these water-based polymers have a limited 
range of safe voltages of 0 to approximately 1.7 volts that can be applied before electrolysis occurs, which generates large bubbles on the polymer surface and can damage the polymer's performance over time [23].

\subsubsection{Experimental Facilities and Setup}

A millimeter-scale channel was constructed to study how vibrating ionic polymers can impact a laminar incident flow. The width and height of the test section had nominal dimensions of 25.5 millimeters and 13 millimeters, and that channel length was $450 \mathrm{~mm}$. A schematic showing the flow channel is illustrated in Figure 2.3. Pressure-driven flow in this channel was generated using a KD Scientific model 230 syringe pump accurate to $+/-1 \%$, and the working fluid of the channel was chosen to be water. All experiments were run at a Reynolds number of 10, which corresponds to a mean velocity in the test section of $0.7 \mathrm{~mm} / \mathrm{s}$. The entrance length for steady laminar flow in a duct may be calculated using the formula [24]

$$
\frac{l_{e}}{D} \approx 0.06 R e+0.5
$$

where $l_{e}$ is the entrance length, $D$ is the diameter of the channel, and $R e$ is the Reynolds number. Therefore, for a Reynolds number of 10 , the ratio $l_{e} / D$ is 1.1 . Since the experiment is in a rectangular channel, $D$ is calculated as $D_{h}$, the hydraulic diameter, which is

$$
D_{h}=\frac{4 A}{P}
$$

where $A$ is the channel cross sectional area and $P$ is its perimeter. For this set up, the hydraulic diameter is $17 \mathrm{~mm}$. Using Equations 1 and 2, the flow is calculated to be fully developed at $19 \mathrm{~mm}$ downstream from the entrance of the device. For additional assurance that the flow was fully developed, the polymers were placed approximately 
$200 \mathrm{~mm}$ downstream of the channel entrance. A summary of the relevant experimental dimensionless parameters is shown in Table 2.1. The calculated Reynolds number is 10, with a Peclet number of nearly $1.4 * 10^{6}$. Both the Reynolds and Peclet numbers confirm that this flow is highly laminar in nature and that diffusion alone will not be a reliable means for mixing. Also, the Reynolds number based on polymer vibration is 0.17 , indicating that for this experiment; the inertia of the incident flow is much higher than that of the polymer actuation.

Table 2.1 A summary of relevant dimensionless parameters.

\begin{tabular}{|c|c|c|}
\hline $\begin{array}{c}\text { Channel Reynolds } \\
\text { Number, Re }\end{array}$ & $R e_{c}=\frac{U D_{h}}{v}$ & $R e_{c}=\frac{0.0007 \mathrm{~m} / \mathrm{s} * 0.017 \mathrm{~m}}{1.15 * 10^{-6} \mathrm{~m}^{2} / \mathrm{s}}=10$ \\
\hline $\begin{array}{c}\text { Polymer-based Reynolds } \\
\text { Number, Re }\end{array}$ & $R e_{p}=\frac{U_{T} A_{T}}{v}$ & $R e_{p}=\frac{0.002 \mathrm{~m} / \mathrm{s} * 0.001 \mathrm{~m}}{1.15 * 10^{-6} \mathrm{~m}^{2} / \mathrm{s}}=0.17$ \\
\hline Peclet Number & $P e=\frac{U l}{D_{i f f}}$ & $P e=\frac{0.0007 \mathrm{~m} / \mathrm{s} * .450 \mathrm{~m}}{0.2272 * 10^{-9}}=1.38 * 10^{6}$ \\
\hline
\end{tabular}

For polymer actuation, the polymer was supplied a $1.5 \mathrm{~V}$ amplitude square wave generated using an HP power supply. Since this signal had negligible current, the square wave was first run through a current amplifier to supply the current needed to run the ionic polymers, which is approximately $200 \mathrm{~mA}$. The square wave signal had an offset of zero to alternatively send positive and negative voltages to the polymer. The change of polarity of voltage is necessary to reverse the direction of the ion attraction and cause the polymer to bend to the opposite direction. A schematic illustrating this concept is shown in Figure 2.4. 


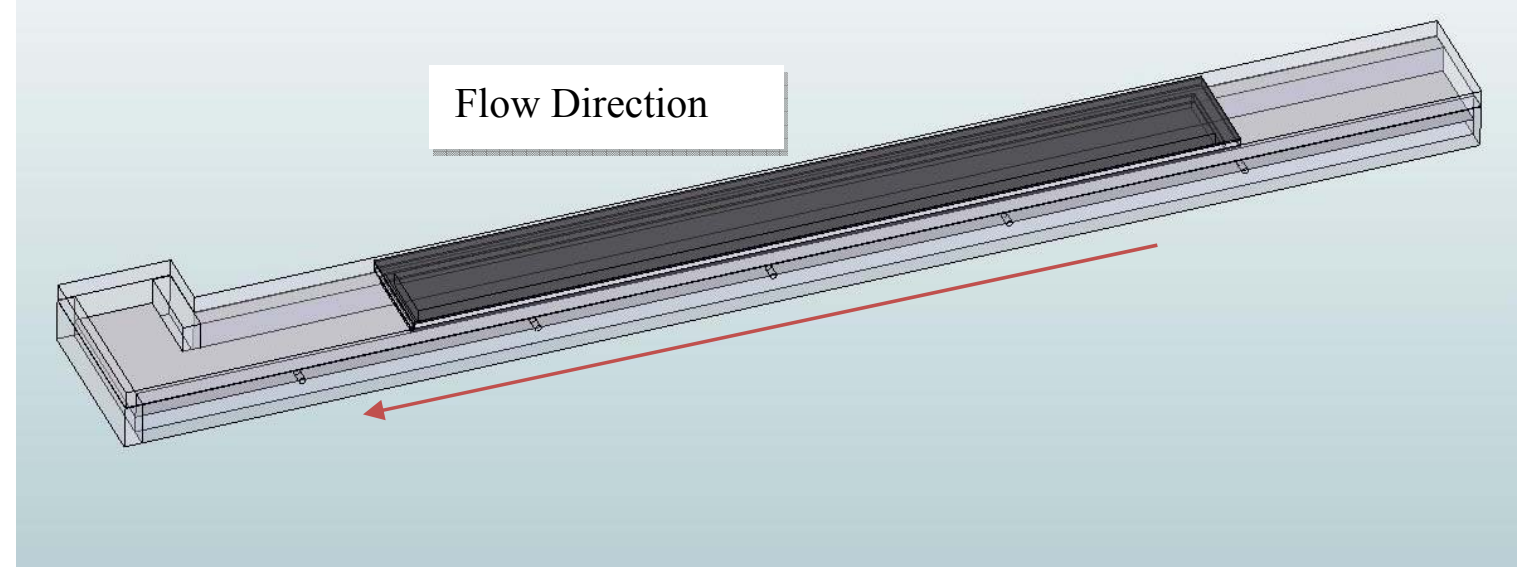

Figure 2.3. Channel used for mixing polymer testing. The top of the channel (shown in black) is removable to allow for placement of multiple polymer configurations.

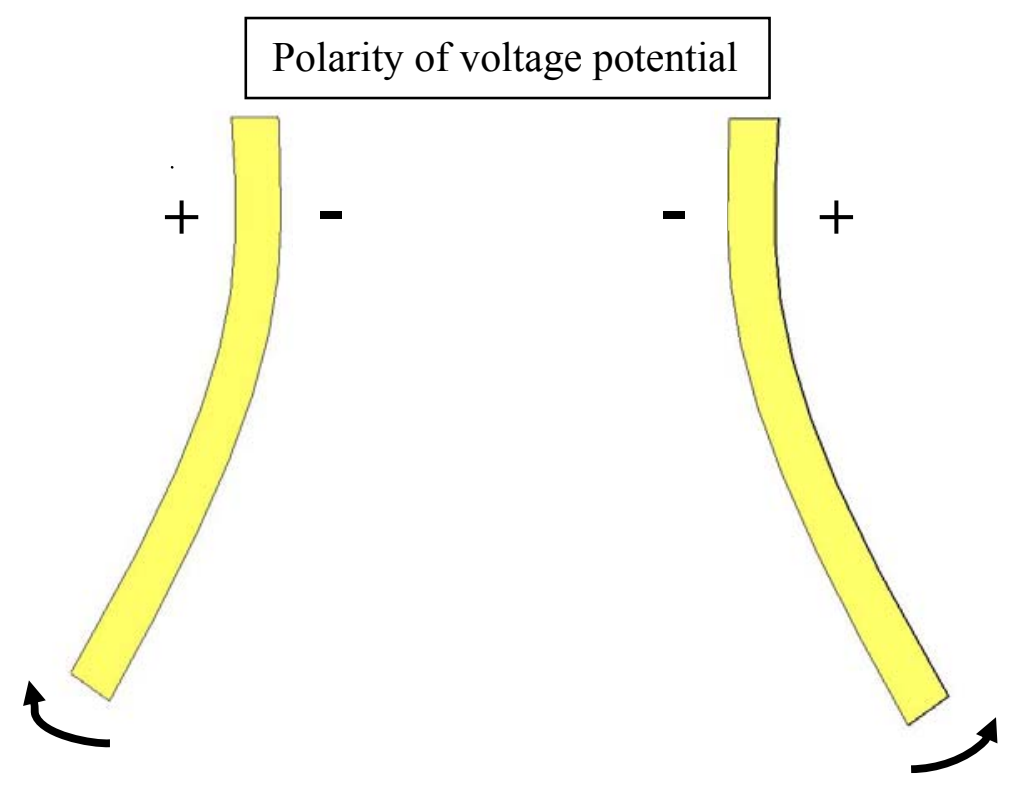

Figure 2.4. Schematic showing how ionic polymers deflect with respect to changes in voltage polarity. 


\subsubsection{Experimental Setup}

Both spatial and temporal characteristics of the flow are important to develop intuition as to how the polymer impacts the flow field. Therefore, a DPIV investigation of the flow within the channel was performed, with the laser plane positioned at several locations along the channel downstream of the polymers. DPIV is a mature optical flow measurement technique in which a coherent light source is expanded into a thin plane, and then is directed across a specific region of interest within the flow. The flow is seeded with neutrally-buoyant flow tracers which follow the flow path. High speed cameras are used to capture the motion of these flow tracer particles, and the digital photos taken with the camera are used in conjunction with FFT-based cross correlation techniques to obtain planes of velocity vectors from particle displacements between frames. Some of the earliest work in DPIV development was performed by Adrian [25, 26] and Westerweel [27]. Errors in our DPIV experiments are small, on the order of 0.1 pixels, whereas a typical pixel displacement of a flow tracer between correlated frames is 2-4 pixels [28].

The Regions-Of-Interest (ROI) at which the laser plane was located were both streamwise to the incident flow as well as orthogonal to the mean flow direction. The streamwise flow data was taken merely to ensure the flow quality in the channel. Schematics of both set ups with the relative locations of the camera and laser plane are shown in Figure 2.5 and Figure 2.6. For all data, the sampling frequency was $128 \mathrm{~Hz}$, with the magnification of the high speed IDT X-5 CMOS camera being 22 microns/pixel. Because of the small velocities in the test section, pixel displacements between consecutive frames were expected to be small, and single-pulsed DPIV was used. The image size was 1136 pixels by 604 pixels, corresponding to the channel width of 25.5 $\mathrm{mm}$ and channel height of $13 \mathrm{~mm}$. Flow tracers for this configuration were selected to be 10 micron neutrally-buoyant polystyrene spheres. 

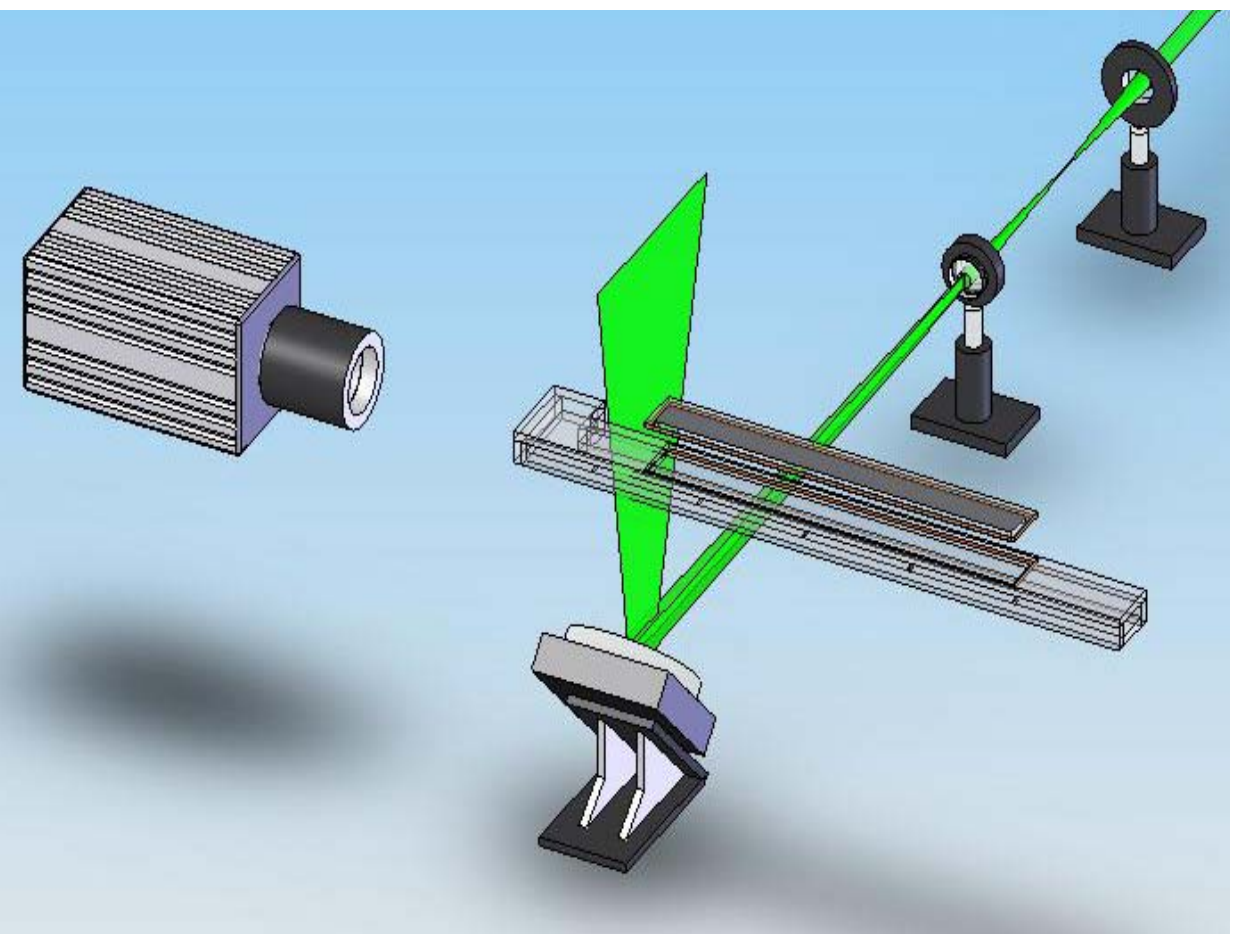

Figure 2.5. DPIV configuration for orthogonal ROI testing to obtain flow structures in the cross section of the channel.

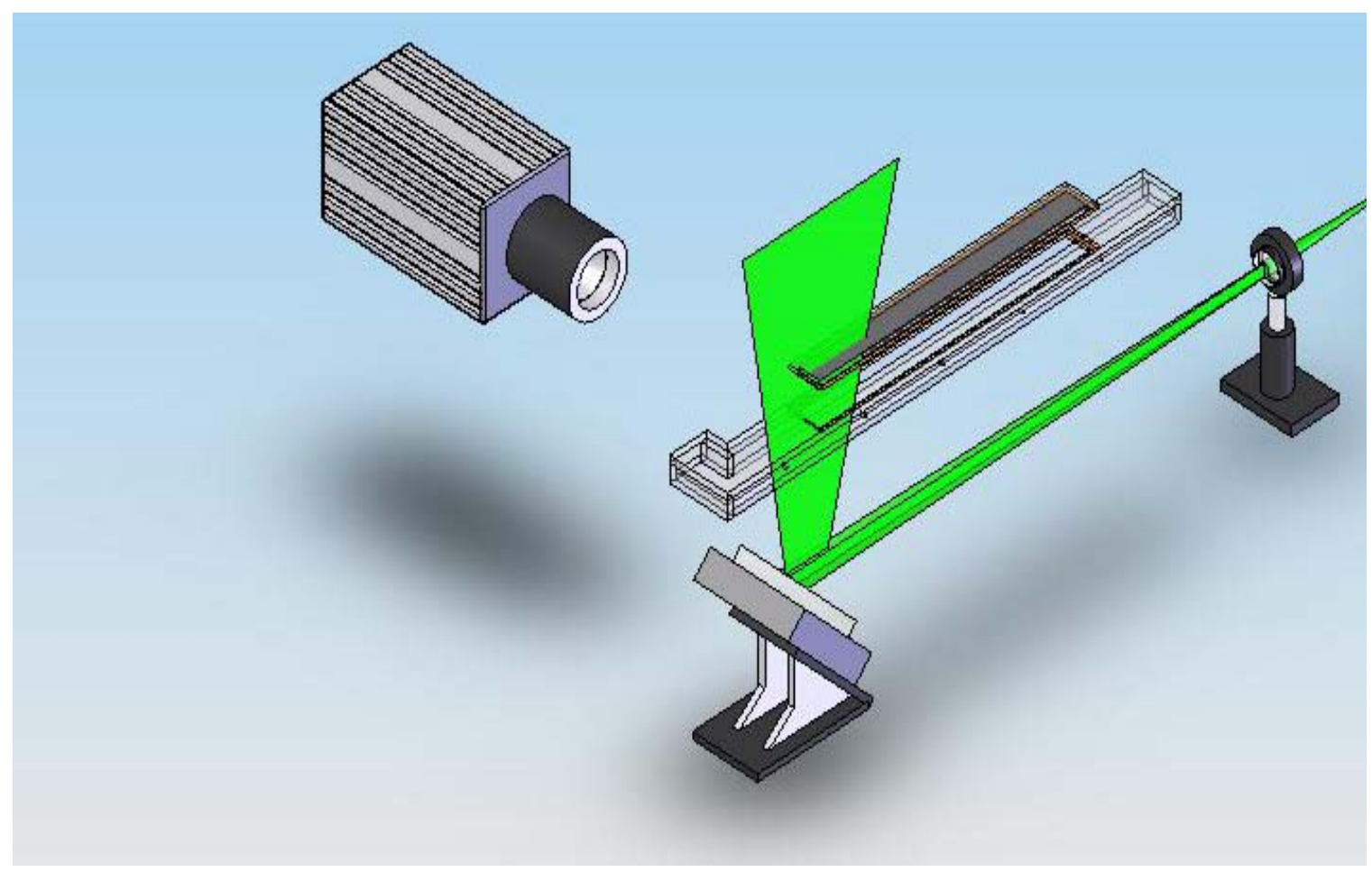

Figure 2.6. DPIV configuration for streamwise flow data acquisition. The laser plane was located at the channel centerline. 
Both one and four polymer arrays were tested in different configurations to determine the impact on the flow. The polymers were all cut to be approximately $6.5 \mathrm{~mm}$ long, with a width of $8.5 \mathrm{~mm}$. Physically, this corresponds to a half-channel height by a third of the channel width. A schematic showing the locations of these polymers relative to the channel for two four-polymer configurations are illustrated in Figure 2.7. The polymers in these configurations were separated by one channel width.

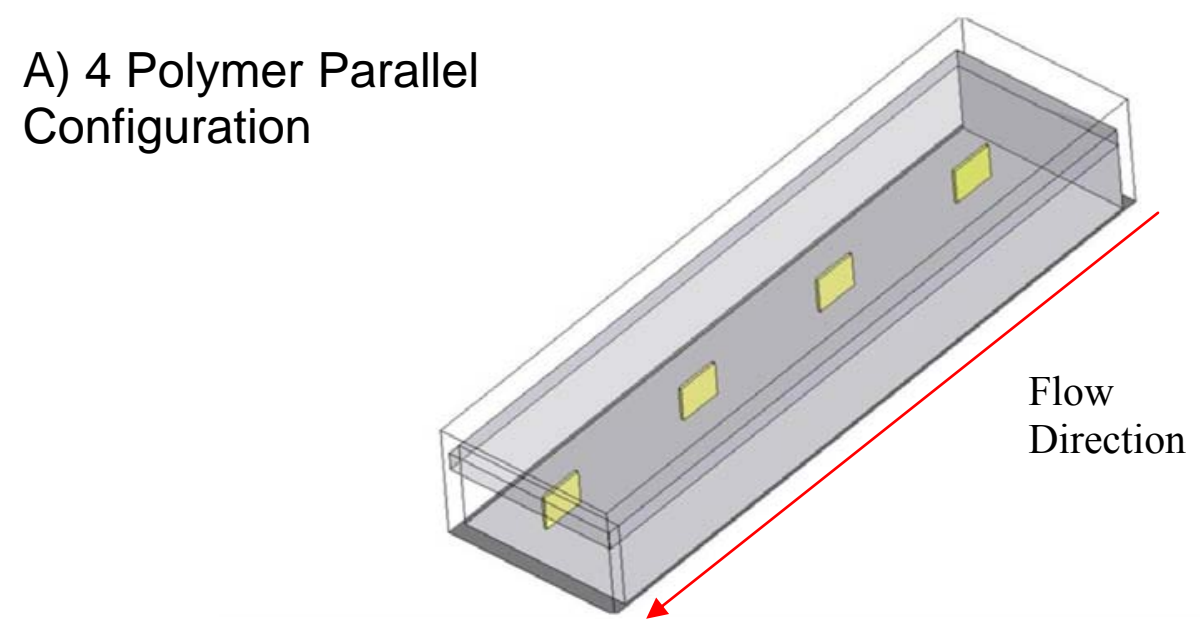

\section{B) 4 Polymer Orthogonal} Configuration

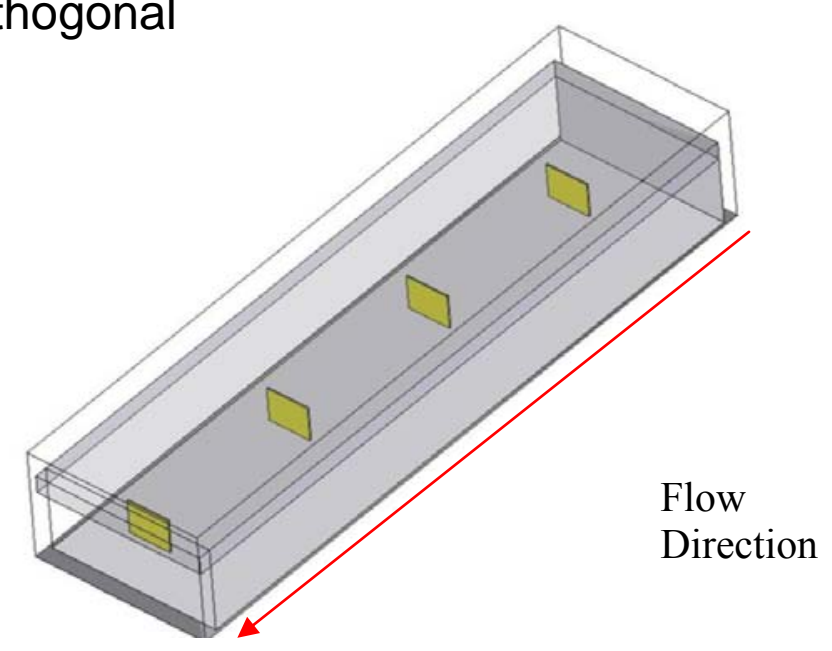

Figure 2.7. Mixing polymer configurations. A) Shows a four polymer configuration with polymers in the "parallel" orientation. B) Shows a four polymer configuration with polymers in the "orthogonal" orientation. 
An additional four polymer configuration was tested in which the polymer cantilever orientations alternated between orthogonally-oriented and parallel-oriented polymers (a four polymer configuration with two parallel polymers and two orthogonal polymers). In terms of the spacing between the polymers, all cases were tested at a spacing of one channel width. This corresponds to approximately 3 cantilever heights between polymer actuators. The single polymer configurations were tested in the same orientations, using the most downstream polymer for testing of these orientations. For all configurations a complete test matrix is given in Table 2.2.

Table 2.2. Test matrix for a single ensemble of polymer testing.

\begin{tabular}{|c|c|c|c|c|c|}
\hline Case & Polymer Orientation & $\begin{array}{l}\text { Number of } \\
\text { Polymer } \\
\text { Actuators }\end{array}$ & $\begin{array}{l}\text { State of } \\
\text { Polymer(s) }\end{array}$ & $\begin{array}{l}\text { Plane location } \\
\text { relative to } \\
\text { polymer, } \mathrm{mm}\end{array}$ & $\begin{array}{l}\text { Region of Interest } \\
\text { Orientation }\end{array}$ \\
\hline 1 & Parallel to Flow & 1 & Actuation & 0 & Cross-Sectional \\
\hline 2 & Parallel to Flow & 1 & Baseline & 0 & Cross-Sectional \\
\hline 3 & Parallel to Flow & 1 & Actuation & 5 & Cross-Sectional \\
\hline 4 & Parallel to Flow & 1 & Baseline & 5 & Cross-Sectional \\
\hline 5 & Parallel to Flow & 1 & Actuation & 10 & Cross-Sectional \\
\hline 6 & Parallel to Flow & 1 & Baseline & 10 & Cross-Sectional \\
\hline 7 & Orthogonal to Flow & 1 & Actuation & 0 & Cross-Sectional \\
\hline 8 & Orthogonal to Flow & 1 & Baseline & 0 & Cross-Sectional \\
\hline 9 & Orthogonal to Flow & 1 & Actuation & 5 & Cross-Sectional \\
\hline 10 & Orthogonal to Flow & 1 & Baseline & 5 & Cross-Sectional \\
\hline 11 & Orthogonal to Flow & 1 & Actuation & 10 & Cross-Sectional \\
\hline 12 & Orthogonal to Flow & 1 & Baseline & 10 & Cross-Sectional \\
\hline 13 & Orthogonal to Flow & 4 & Actuation & 10 & Cross-Sectional \\
\hline 14 & Orthogonal to Flow & 4 & Baseline & 10 & Cross-Sectional \\
\hline 15 & Orthogonal to Flow & 4 & Actuation & 5 & Cross-Sectional \\
\hline 16 & Orthogonal to Flow & 4 & Baseline & 5 & Cross-Sectional \\
\hline 17 & Parallel to Flow & 4 & Actuation & 10 & Cross-Sectional \\
\hline 18 & Parallel to Flow & 4 & Baseline & 10 & Cross-Sectional \\
\hline 19 & Parallel to Flow & 4 & Actuation & 5 & Cross-Sectional \\
\hline 20 & Parallel to Flow & 4 & Baseline & 5 & Cross-Sectional \\
\hline 21 & Alternatina Orientations & 4 & Actuation & 10 & Cross-Sectional \\
\hline 22 & Alternatina Orientations & 4 & Baseline & 10 & Cross-Sectional \\
\hline 23 & Alternatina Orientations & 4 & Actuation & 5 & Cross-Sectional \\
\hline 24 & Alternatina Orientations & 4 & Baseline & 5 & Cross-Sectional \\
\hline 27 & Orthogonal to Flow & 4 & Actuation & 5 & Streamwise \\
\hline 28 & Orthogonal to Flow & 4 & Baseline & 5 & Streamwise \\
\hline 31 & Parallel to Flow & 4 & Actuation & 5 & Streamwise \\
\hline 32 & Parallel to Flow & 4 & Baseline & 5 & Streamwise \\
\hline 35 & Alternatina Orientations & 4 & Actuation & 5 & Streamwise \\
\hline 36 & Alternating Orientations & 4 & Baseline & 5 & Streamwise \\
\hline
\end{tabular}


For the orthogonal ROI, either two or three planes of data were acquired for each configuration tested. For the single cantilevered polymer configurations, three planes were located 0,5 , and $10 \mathrm{~mm}$ downstream from the trailing edge of the cantilevered polymer, where the polymer was aligned either parallel or orthogonal with respect to the streamwise centerline of the test section. For the three different four-polymer configurations tested, the orthogonal ROIs were located at 5 and $10 \mathrm{~mm}$ further downstream of the last actuating polymer in the configuration. This corresponds to approximately one and two cantilever heights downstream. The objective in taking data at the trailing edges of the single polymers was to capture the additional flow structures resulting from actuation and to directly visualize the impact on the flow.

\subsubsection{Mixing Quantification}

Since we have obtained a two-dimensional field of velocities from the DPIV measurements, this information may be used to derive secondary information about the flow field. Since the objective of using the ionic polymers is to create a mixing device, the ability to quantify how much mixing occurs in the channel is of particular interest. Many different mixing metrics are available, but few that can be used directly for experimental work are based directly off quantitative velocity data. Some groups have used the magnitude of the rate of strain tensor as a basis for defining mixing potential in laminar flows $[29,30]$. The rate of strain tensor, $e$, is calculated by:

$$
e_{i j}=\frac{1}{2}\left(\nabla U+\nabla U^{T}\right)
$$

where $U$ is the total velocity field. For a two-dimensional flow field like that obtained from DPIV data, the three-dimensional rate of strain tensor reduces to:

$$
e_{i j}=\left[\begin{array}{cc}
\frac{\partial w}{\partial z} & \frac{1}{2}\left(\frac{\partial w}{\partial y}+\frac{\partial v}{\partial z}\right) \\
\frac{1}{2}\left(\frac{\partial v}{\partial z}+\frac{\partial w}{\partial y}\right) & \frac{\partial v}{\partial y}
\end{array}\right]
$$


where $w$ and $v$ refer to the velocities in the $z$ and $y$ direction, respectively. This tensor may be directly calculated from the velocity data reduced from the raw experimental frames. For this experiment, the $y$ and $z$ coordinates were normalized with respect to the hydraulic diameter, $D_{h}$. The $w$ and $v$ velocities were normalized with respect to the streamwise velocity, $U$. The rate of deformation tensor, $\xi$, (which for this twodimensional case is a tensor of order 0 and is a scalar) is found from the magnitude of the rate of strain tensor, written as

$$
\xi=\left|e_{i j}: e_{i j}\right|
$$

This scalar field describes the maximum possible creation of interfaces within the flow, and provides an estimate of this quantity since the full rate of strain tensor components cannot be calculated. The creation of many interfaces allows for an improved diffusion speed for mixing the overall flow, since the presence of more interfaces creates smaller regions over which diffusion can act. However, this metric merely provides a local estimate of the mixing potential, which does not indicate whether the bulk flow is mixed throughout either the channel or, in the case of this experiment, the plane of interest. A code was written in Matlab to perform this calculation for each measurement point in the flow field for the time-resolved data obtained for each case. Second order central finite differences were used to calculate the derivatives of the velocity field. Considering the velocity measurement error previously reported, the estimated uncertainty associated with the calculation of the strain rate tensor is on the order of $20 \%$.

\subsection{Experimental Results}

The results of the DPIV experiment are presented in this section. First, processed data taken with flow in the channel with no polymers present is discussed. Then data taken in the presence of the polymers with and without actuation and with the same orthogonal laser plane orientation will be presented. 


\subsubsection{DPIV Post Processing and Data Nomenclature}

The raw frames were processed using an FFT-based discrete window offset algorithm with two passes of 32 by 32 pixel windows; with spatial resolution of one vector every 8 pixels. This resulted in a reduced flow field of approximately 9800 vectors. Because pixel displacements between frames were very small, based on the initial sampling of $128 \mathrm{~Hz}$, the data was effectively downsampled to $32 \mathrm{~Hz}$, since $128 \mathrm{~Hz}$ sampling resolution could not provide acceptable accuracy due to the low pixel displacement between consecutive frames.

The spatial coordinates are normalized using the hydraulic diameter of the channel. The coordinates $z / D_{h}$ and $y / D_{h}$ of zero are selected to be at the mid-channel width and height, respectively. For parallel-oriented cases, the polymers were placed at a location $z / D_{h}$ of zero. For orthogonally-oriented cases, the polymers were positioned in the channel so that they were ideally centered about $z / D_{h}$ of zero. Presenting the results for all cases would be prohibitively long; instead only representative cases which demonstrate the effect due to polymer actuation will be presented.

\subsubsection{Results for Selected Polymer Configurations}

Data taken to characterize the cross section of the flow downstream of the polymers shows that differences appear in the flow field between the baseline and actuation cases for several different configurations of cantilevered polymers. The differences in the velocity fields between baseline and actuation cases are of principal interest, since the presence of the polymer cantilevers in the channel even with no actuation disturbs and redirects the flow. In essence, polymers at rest act as obstructions to create secondary flows for mixing, similar to some of the examples presented in the introduction.

To collapse the data in a way so that the polymer impact can be readily visualized, the time-averaged percent difference in velocity magnitude between baseline and actuation cases is calculated. This percent difference will be defined as 


$$
P_{\text {Diff }}=\frac{V_{\text {actuation }}-V_{\text {baseline }}}{V_{\text {baseline }}} * 100
$$

where $V_{\text {actuation }}$ is the time-averaged velocity measured during actuation and $V_{\text {baseline }}$ is the time-averaged velocity measured during the baseline case. To obtain these results of each baseline and actuation case, the time-averaged velocity magnitude is first normalized with respect to the average streamwise channel velocity. At each vector location $i, j$, the percent difference is then computed using Equation 7, to obtain a field in which the changes at each point may be visualized. Results from both this and the mixing quantification analysis will be presented in this section. First, results for both one parallel and one orthogonal polymer will be discussed, followed by those for a four polymer configuration with orthogonally-oriented polymers.

One Parallel-Oriented Polymer. For reference, a schematic illustrating the relative orientations of the polymer and the laser plane is shown in Figure 2.8. Post-processed plots showing the normalized velocity magnitude for one parallel polymer at $0 \mathrm{~mm}$ downstream is shown in Figure 2.9 superimposed with the velocity streamlines. The local influence of the parallel polymer can be seen for both baseline and actuation cases near $z / D_{h}$ of zero near the base of the polymer from what it appears as a horseshoe streamwise structure as well as a flow structure in the vicinity of the polymer tip. In addition, the streamlines indicate two counter-rotating vortices on either side of the polymer although they appear asymmetric. Finally, from the streamlines a clear velocity upwash is indicative of three dimensional motions normal to our plane of measurement. 


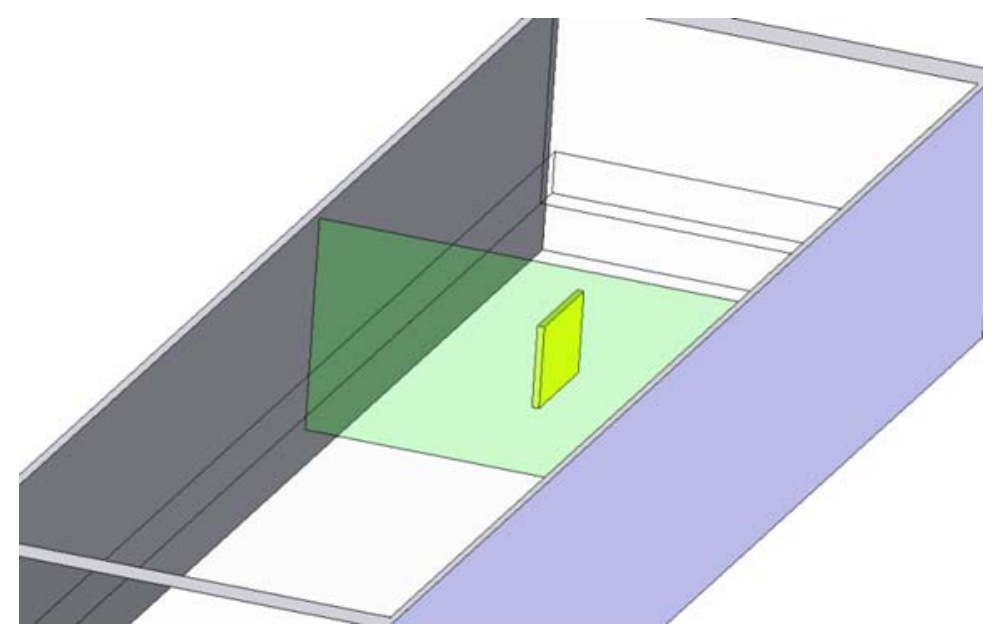

Figure 2.8. Schematic showing the relative location of the parallel-oriented single polymer with respect to the laser plane.

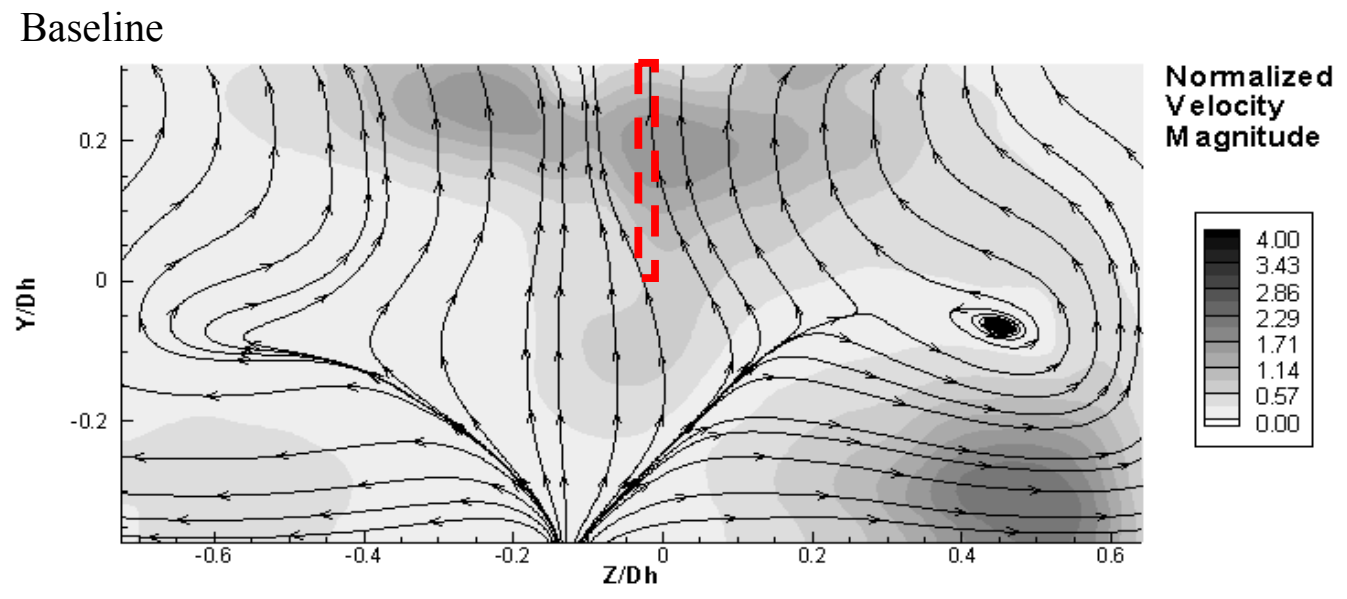

Actuation

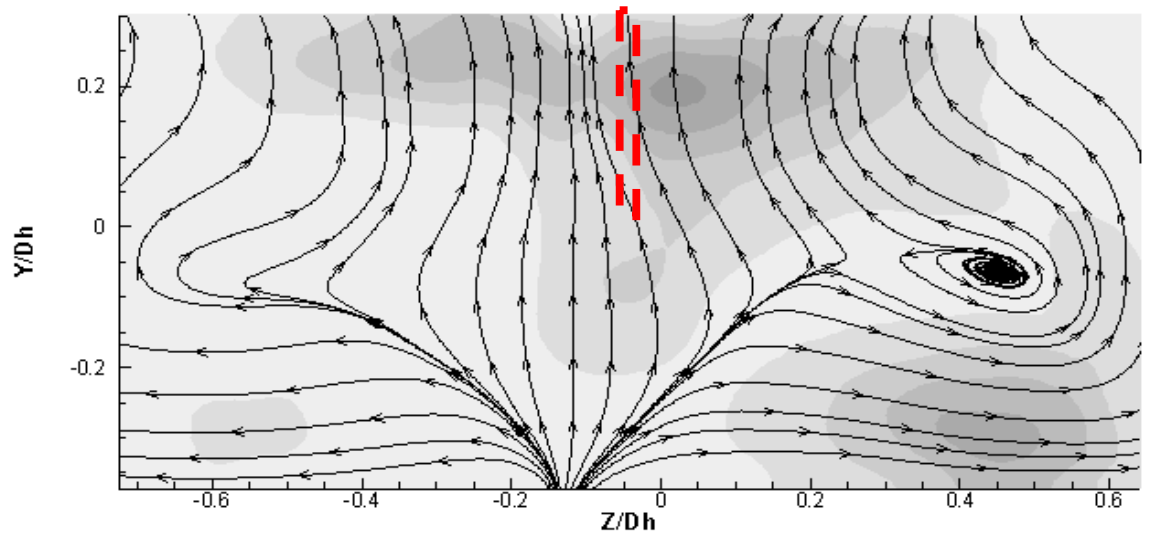

Figure 2.9. Normalized velocity magnitudes for baseline and actuation cases of 1 parallel polymer at its trailing edge. 
Clarification of the effect of the polymer cantilever is seen in the percent difference in normalized velocity magnitudes, which is shown in Figure 2.10. A significant increase, near 200 percent in velocity magnitude appears near $y / D_{h}$ of zero on either side of the approximate tip location of the polymer in Figure 2.10. In this case, the laser plane was located at $0 \mathrm{~mm}$ downstream of the single polymer, which corresponds to its trailing edge. This increase in velocity likely represents the addition of a velocity component in the $z$ direction due to the large displacements at the tip vibration in nearly the same plane in which this data was acquired.
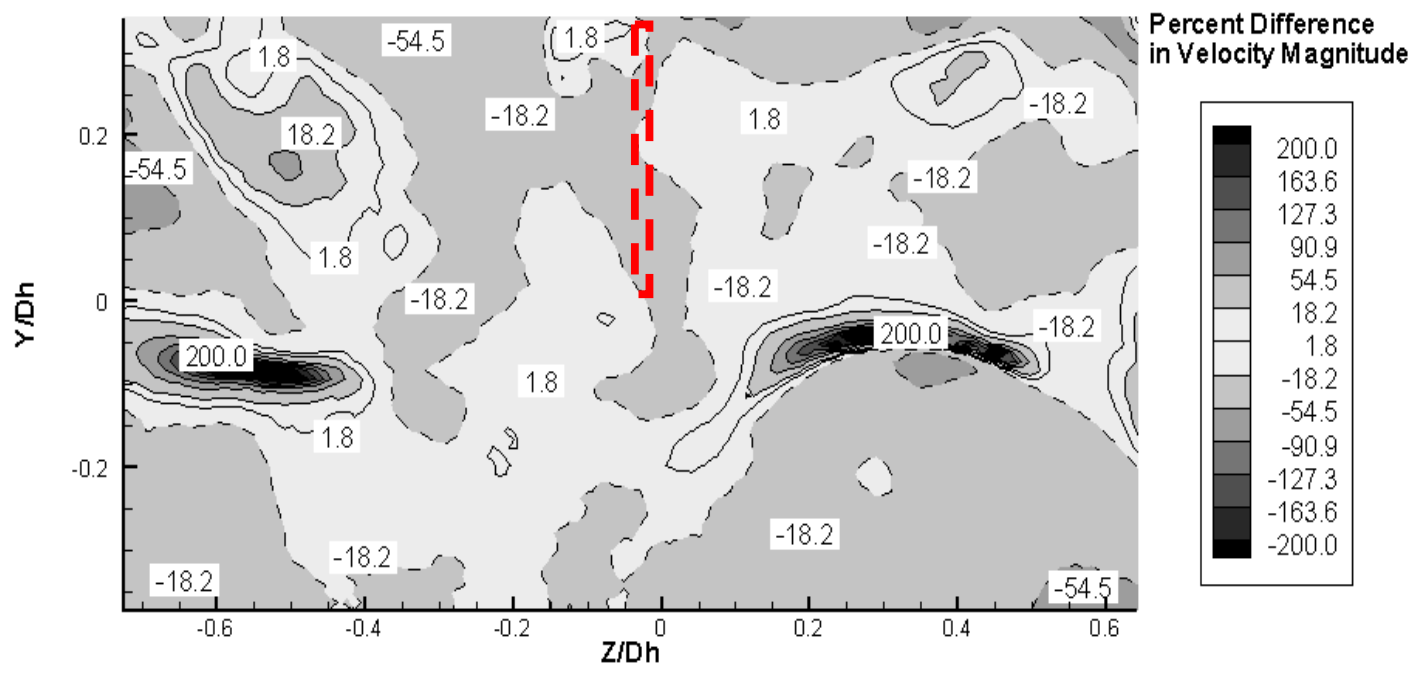

Figure 2.10. Percent difference in velocity magnitude for 1 parallel polymer at $\mathbf{0 ~} \mathbf{m m}$ downstream, or at the cantilevered polymer's trailing edge.

Finally, the magnitude of the strain rate tensor, $\xi$, is shown for both baseline and actuation conditions for this configuration in Figure 2.11, and the percent difference in the two in Figure 2.12. When comparing the two plots shown for the mixing potential, no obvious increases due to actuation appear that could be attributed to the cantilevered polymer. The outline of the parallel-oriented polymer can vaguely be seen in this mixing data contours. Specifically, regions of larger mixing potential than in the overall crosssection appear near $z / D_{h}$ of -0.05 for all positive $y / D_{h}$. This large region of improved mixing also occurs near the top of the channel cross-section, along $y / D h$ of 0.2 . 
Qualitatively, this region occurs in the same locations and magnitudes for both the baseline and actuation case, so we may conclude that this region of increased $\xi$ is not affected by polymer actuation. Examining the percent difference in $\xi$, only a small region near the polymer (shown in dashed lines) shows improvement of $75 \%\left(z / D_{h}\right.$ of -0.1 and $y / D_{h}$ of 0$)$. This increase in the actuation case is expected due to the in-plane vibration of the polymer, and the subsequent energy addition to the flow. Another localized increase in $\xi$ of over 100 percent of actuation over baseline at $z / D_{h}$ of 0 and $y / D_{h}$ of 0.2 , which corresponds to a location along the polymer of about half the cantilever length. Although this localized increase corresponds to polymer actuation, higher $\xi$ near the polymer tip would be expected as opposed to halfway along its length.

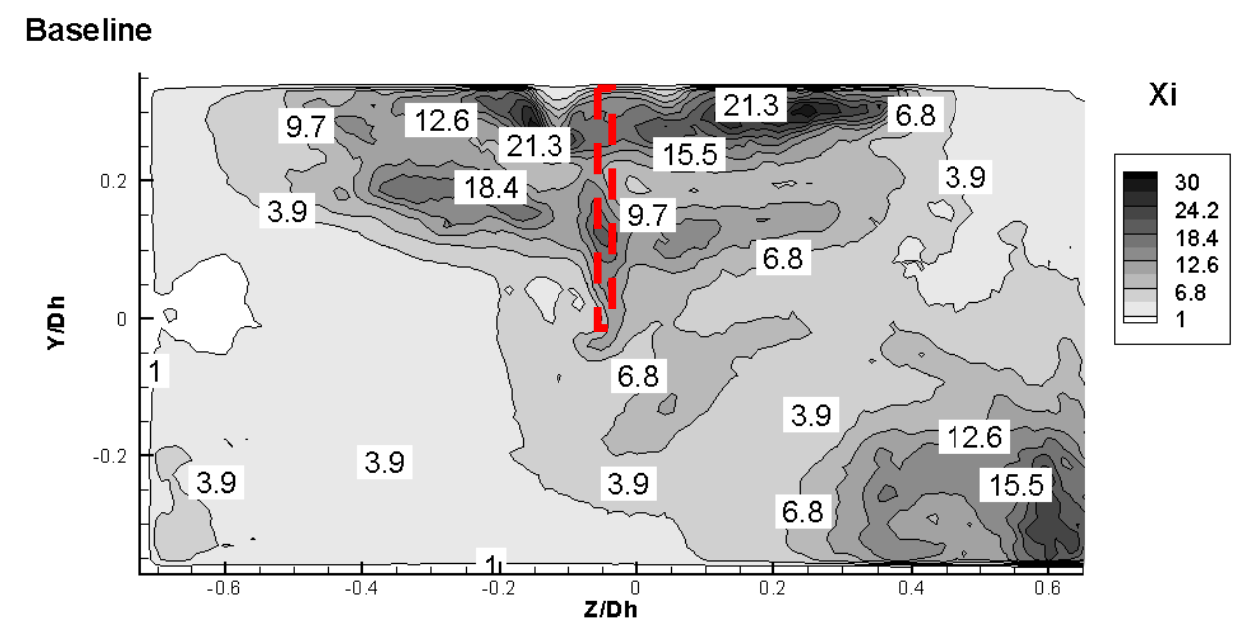

Actuation

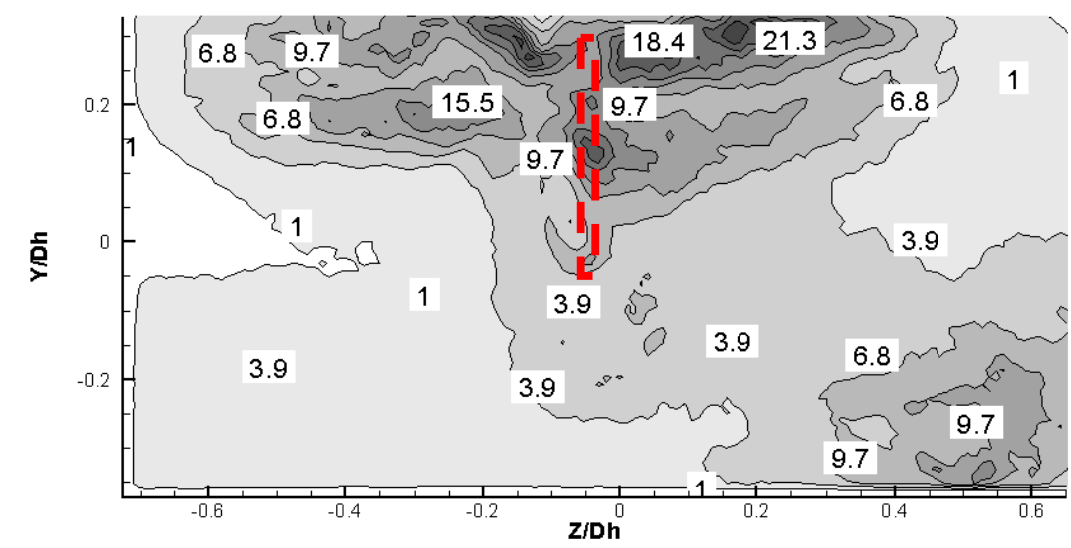

Figure 2.11. Mixing potential, $\xi$, for 1 parallel-oriented polymer at $0 \mathrm{~mm}$ downstream. 


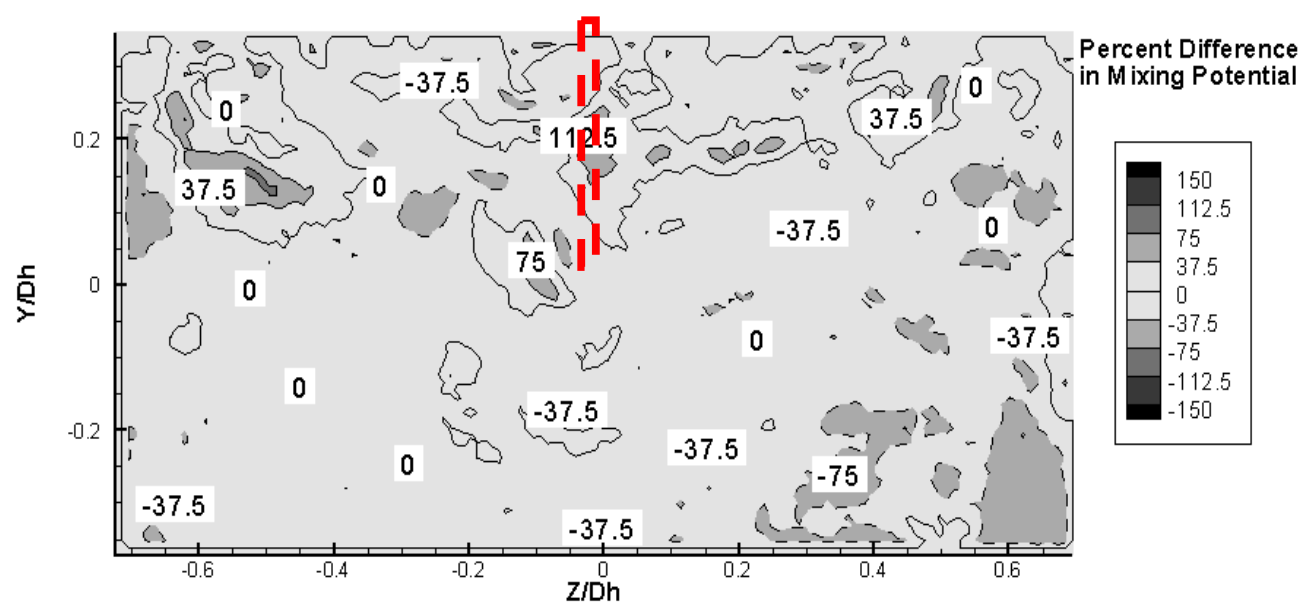

Figure 2.12. Difference in mixing potential, $\xi$, for one parallel-oriented polymer at its trailing edge.

One Orthogonally-Oriented Polymer. A schematic showing the orientation of the orthogonallyoriented ionic polymer compared with the laser plane is shown in

Figure 2.13. Data was taken at 0,5 , and $10 \mathrm{~mm}$ downstream of the polymer, but mainly the $0 \mathrm{~mm}$ or trailing edge case will be presented here. For data taken at the polymer trailing edge, a comparison of the velocity magnitudes is seen in Figure 2.14. Observing these cases we note for both a strongly asymmetric flow indicating a counter clockwise swirl motion. However, comparing baseline and actuation cases, distinct regions in which velocity deficits occur, particularly at $z / D_{h}$ of 0.5 and $y / D_{h}$ of -0.1 , and also at $z / D_{h}$ of 0.2 and $y / D_{h}$ of 0.2 . However, a clear increase in velocity magnitude occurs at $z / D_{h}$ of -0.4 and $y / D_{h}$ between 0 and 0.2 for the actuation case. The normalized velocity magnitudes in these regions average 0.5 for the actuation case, while the average for the baseline case in this region is 0.25 . Comparing this increase to the polymer outline (shown in dashed lines), the polymer shape is traced by the contour levels corresponding to the increase in velocity magnitude. Therefore, we can conclude that this part of the polymer was creating significant deflections such that the in-plane velocity magnitudes show a localized increase for the actuation case. 


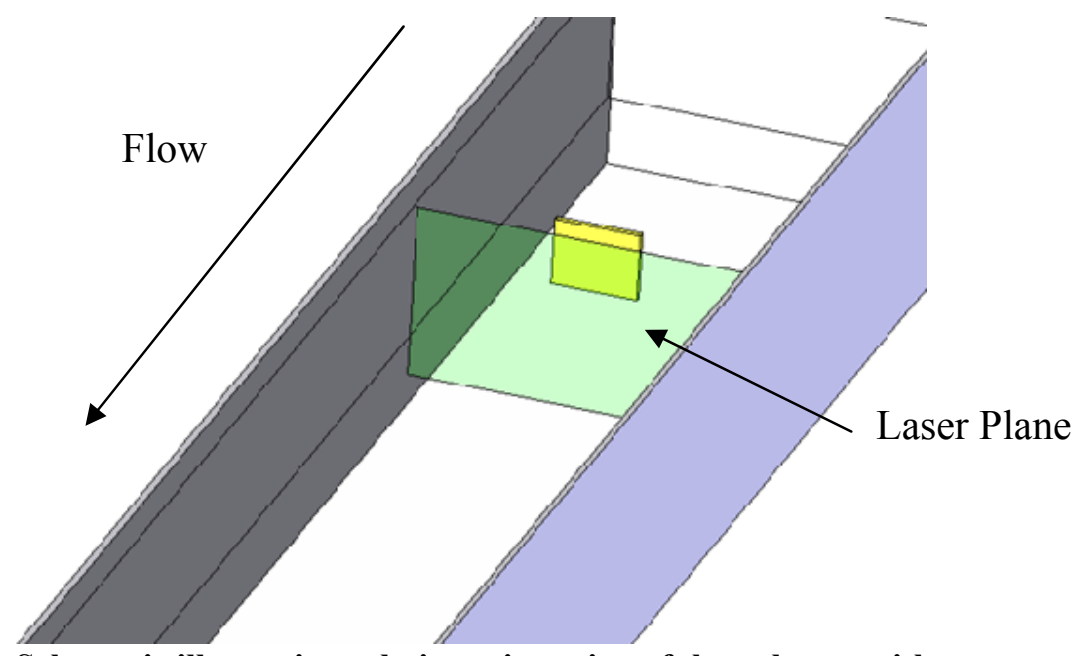

Figure 2.13. Schematic illustrating relative orientation of the polymer with respect to the laser plane
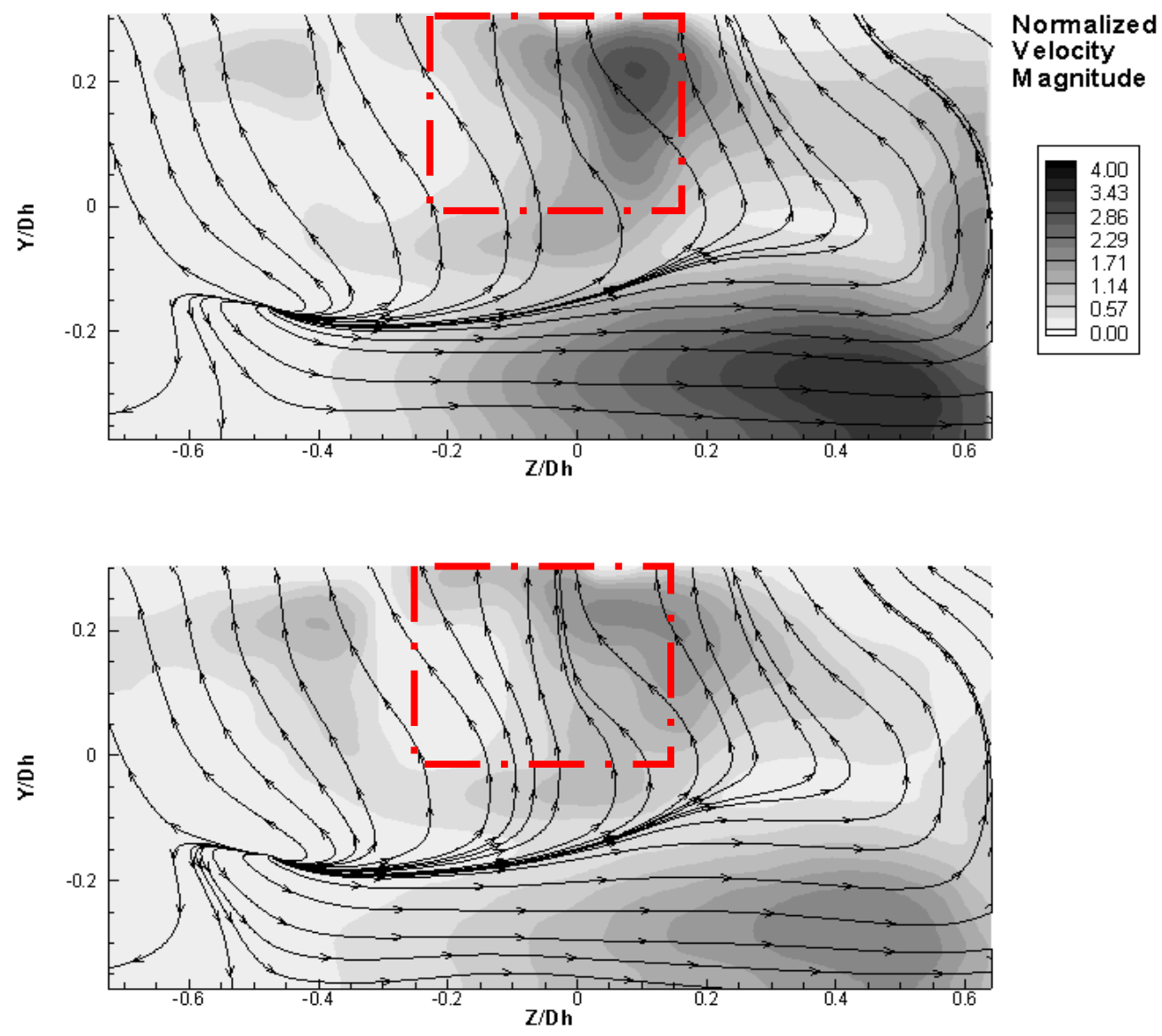

Figure 2.14. Time-averaged baseline and actuation velocity fields normalized by the mean streamwise velocity for one orthogonally-oriented polymer at its trailing edge. 
The percent difference in velocity magnitude for the trailing-edge case for one orthogonally-oriented polymer cantilever is shown in Figure 2.15. The dashed contour lines represent negative values, and the polymer outline is traced by a dashed line. The average increase in velocity between actuation and baseline is $36 \%$ over the entire crosssection for this particular case. Examining the data for the effect of the polymer actuation, there is a clear localized increase in velocity magnitude near the polymer edge at $z / D_{h}$ of -0.4 and $y / D_{h}$ between 0 and 0.2 for the actuation case, and the significant difference in velocities noted in the velocity magnitude plots in Figure 2.14 are seen here as a large area captured by a contour describing 200 or more percent increase in velocity. However, this significant increase on the left hand side of the polymer does not occur on the right, and this asymmetry is clear in both the percent difference plots and the velocity magnitude plots for this case. This discrepancy could be explained by imperfect orthogonal placement of the cantilevered polymer with respect to the mean flow. This could lead to a strong out-of-plane component of the flow, and could also explain why such a deficit occurred. Another possibility is from non-uniform ionic polymer straining and creating large deflections on one side, while barely differing from the baseline case on the other.

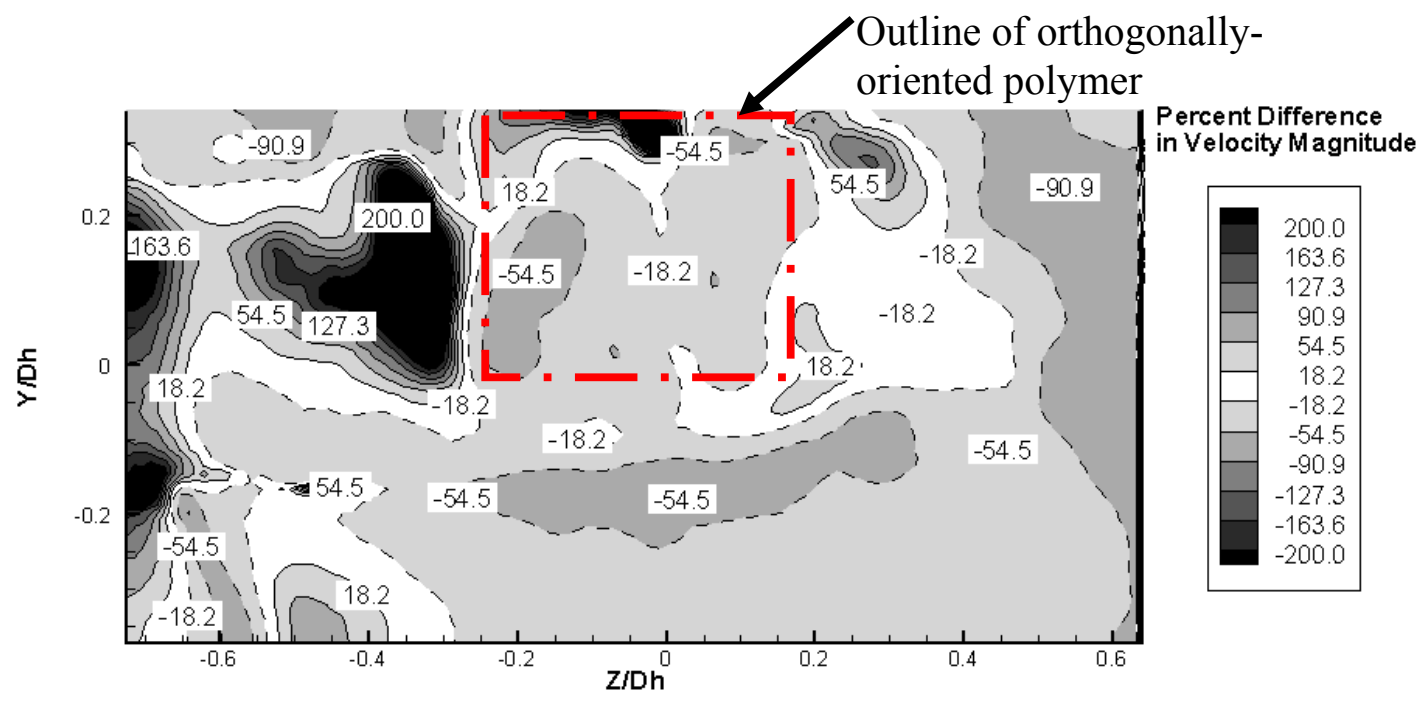

Figure 2.15. One orthogonally-oriented polymer, showing the percent difference in velocity magnitude between baseline and actuation. 
This non-uniform increase is also present when examining the mixing information, shown in Figure 2.16 and Figure 2.17. Although an increase in mixing potential is still visualized in the same region as the largest velocity increase, it appears slightly more displaced away from the polymer's edge. Another feature seen here is a distinct increase in mixing potential that traces the cantilevered polymer's outline on the left hand side near $y / D_{h}$ of zero and $z / D_{h}$ between -0.3 and -0.4 . The increase corresponds to nearly 150 percent increase at $y / D_{h}$ of zero and $z / D_{h}$ of -0.4 . However, as seen in the velocity magnitude plots, no such increase in velocity occurs due to polymer actuation at $y / D_{h}$ of zero and $z / D_{h}$ of $0.2-0.4$, and hence no increases in gradients occur to lead to any difference in the magnitude of the strain rate tensor. Also, from this data, no increase in mixing potential is visualized at the bottom of the polymer away from the polymer edges, at the cantilever tip, located at $y / D_{h}$ of zero. Such an increase likely does not appear in this data due to the vibration of the polymer being out of plane, and therefore would not be recorded based on this data acquisition. 


\section{Baseline}

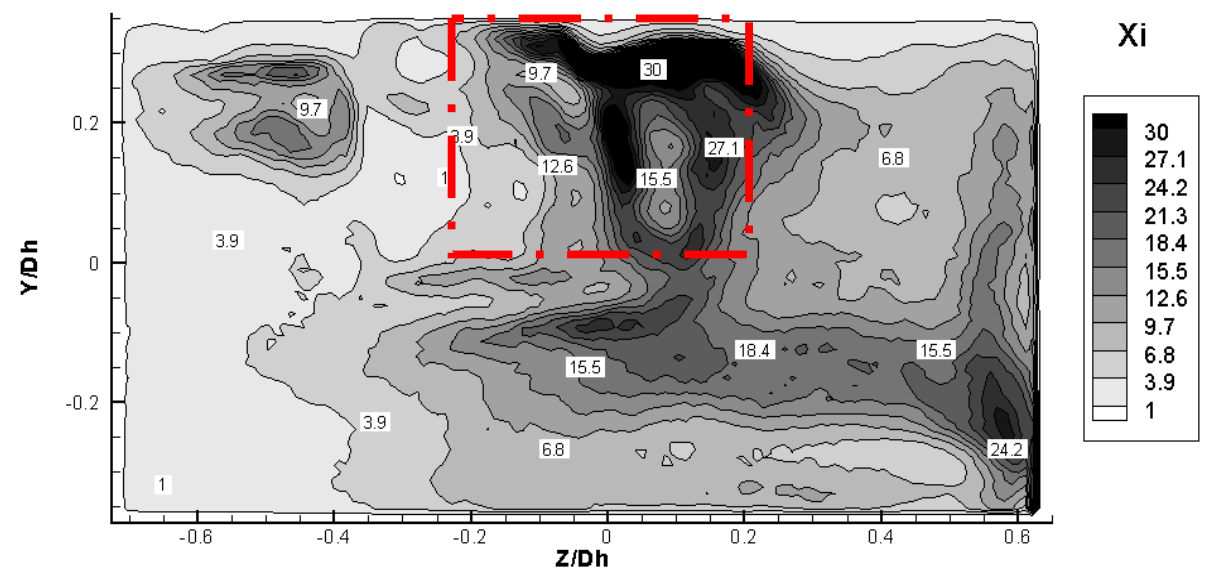

Actuation

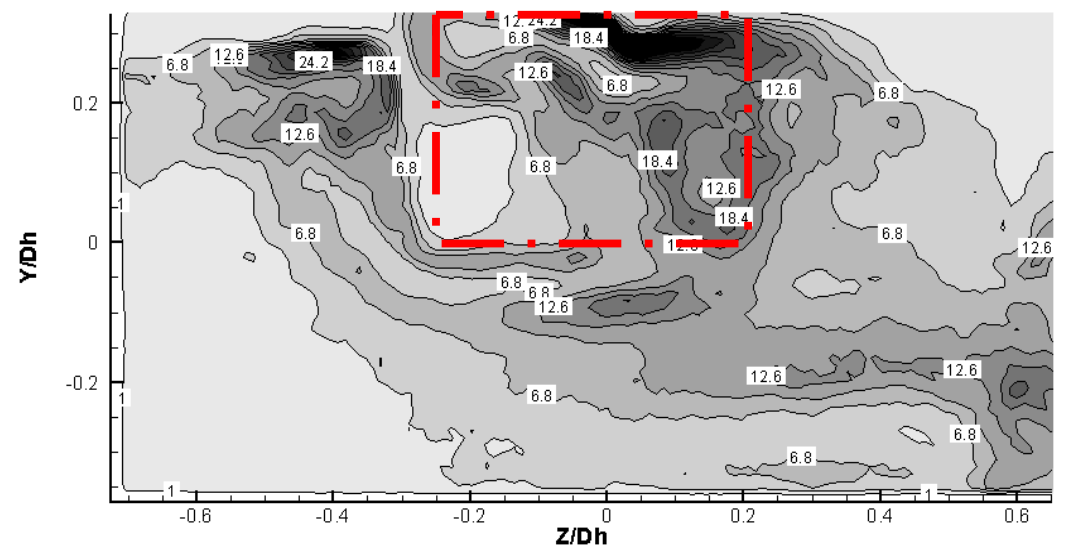

Figure 2.16. Mixing potential, $\mathrm{Xi}$, for one polymer for baseline and actuation with a measurement plane located at the trailing edge of the polymer.

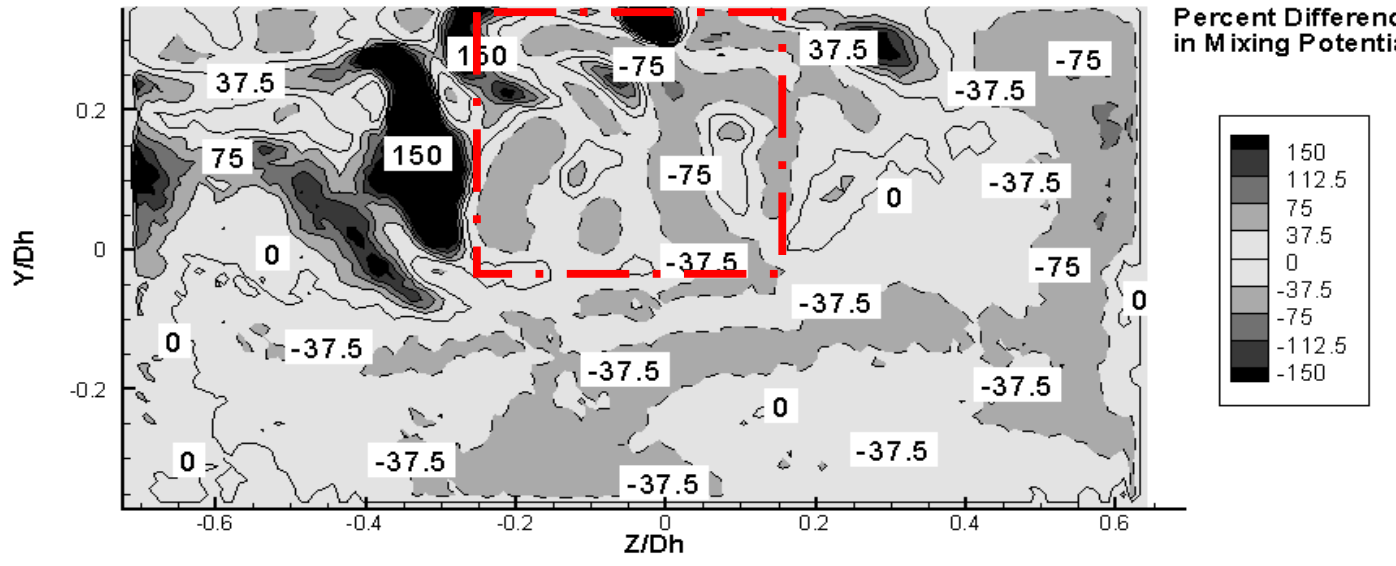

Figure 2.17. Percent difference in the mixing potential, $\xi$, for one orthogonally-oriented polymer at its trailing edge. 
Looking further downstream from the one orthogonal polymer case, in Figure 2.18, the differences that appeared near the polymer at the trailing edge plane are not evident at either 5 or $10 \mathrm{~mm}$ downstream of the polymer. However, for the $5 \mathrm{~mm}$ downstream case, a large region appears where there is an approximately $20 \%$ increase in the velocity magnitude for the actuation case. This is in the region of the bottom edge of the polymer, where the actuating polymer would generate additional vortices than would a polymer at rest. Although the vortical structures are not clearly captured, this increase in velocity physically corresponds to this energy addition.
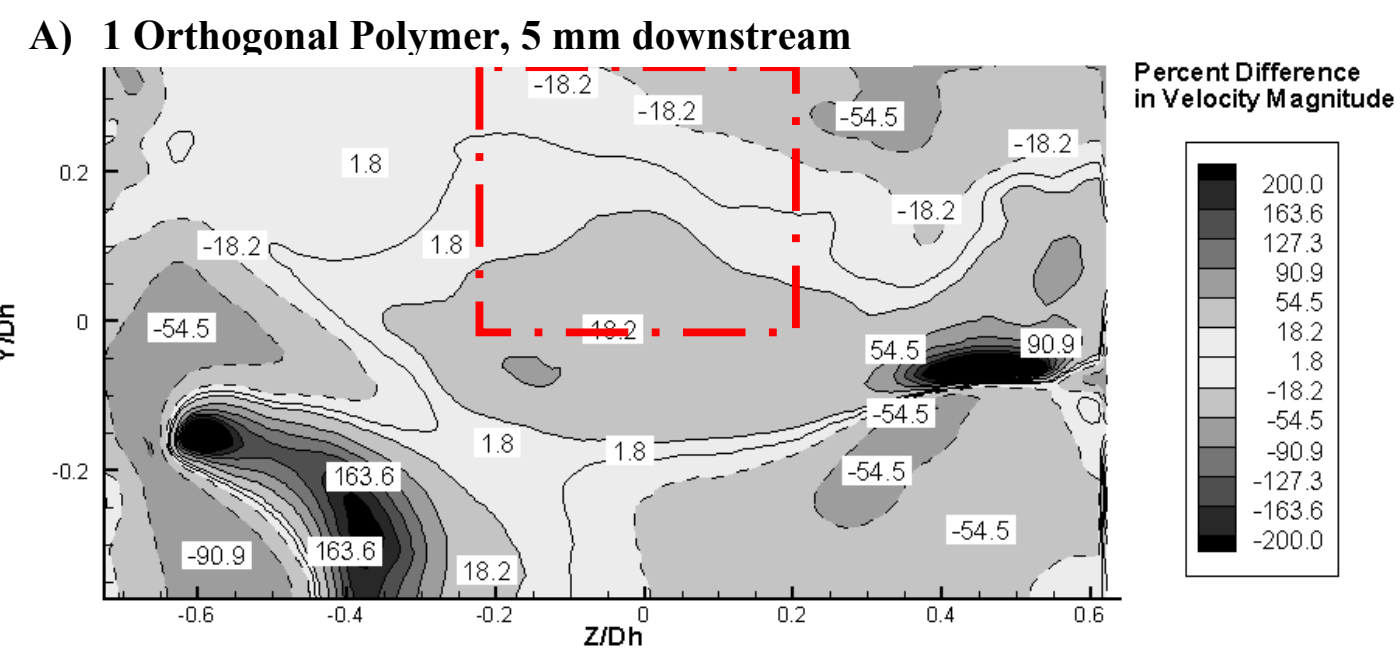

B) 1 Orthogonal Polymer, $10 \mathrm{~mm}$ downstream

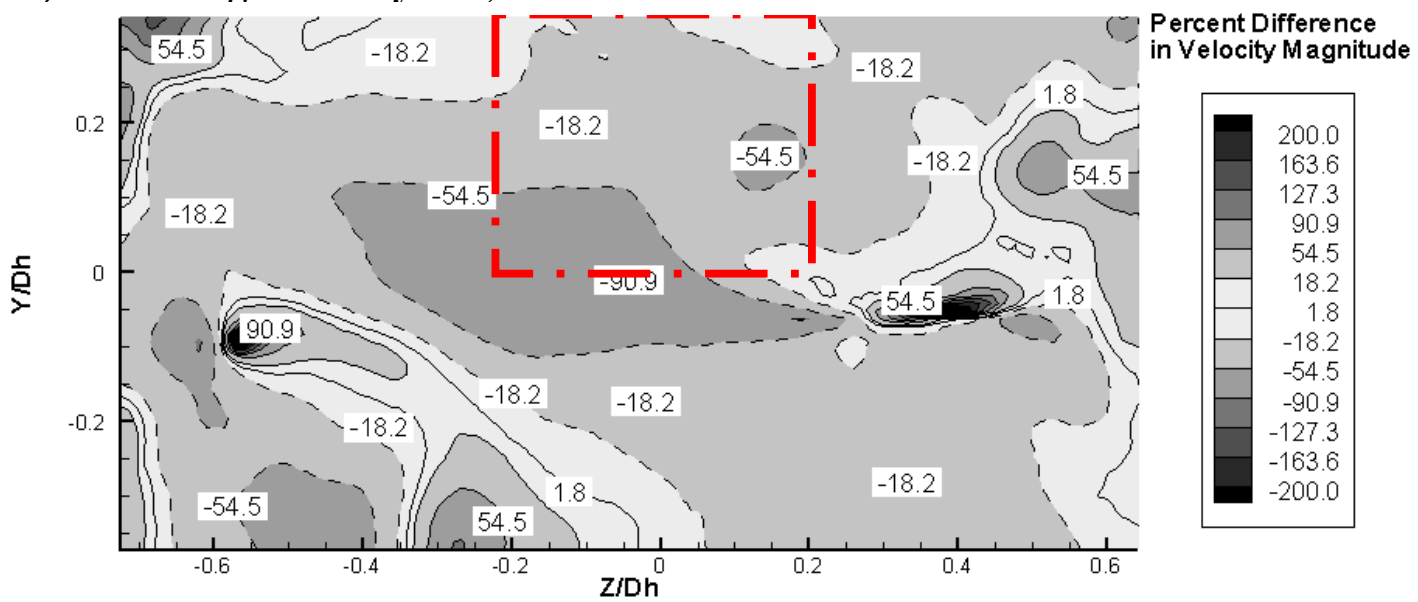

Figure 2.18. Percent difference in velocity magnitude for 1 orthogonally-oriented polymer at 5 and 10 mm downstream. 
At $10 \mathrm{~mm}$ downstream, any clear localized effects very close to the polymer cantilever do not appear. However, comparing the $5 \mathrm{~mm}$ and $10 \mathrm{~mm}$ cases, the large percent difference peaks at $y / D_{h}$ of -0.1 and $z / D_{h}-0.6$ and $y / D_{h}$ of -0.05 and $z / D_{h} 0.4$ translate between the cases. Moreover, the size of these regions is diminished at $10 \mathrm{~mm}$ compared to at $5 \mathrm{~mm}$ as would be expected, since less disturbance would be expected further from the polymer cantilever's surface.

Four Orthogonal Polymers. The time-averaged velocity magnitudes and streamlines for baseline and actuation cases for $5 \mathrm{~mm}$ downstream of the trailing edge of the last cantilevered polymer in this configuration are shown in Figure 2.19. At this location, a clear vortical structure appears in the data, rotating counterclockwise at $z / D_{h}$ of 0.5 . Velocity data for the plane $10 \mathrm{~mm}$ downstream of the last polymer are shown for baseline and actuation cases in Figure 2.20. Here, two counter-rotating vortical structures appear in the data for both the 5 and $10 \mathrm{~mm}$ cases in similar locations. For both the 5 and $10 \mathrm{~mm}$ cases, the ending streamlines indicate out-of-plane motion not captured by DPIV.

For both the $5 \mathrm{~mm}$ and $10 \mathrm{~mm}$ cases, the velocity distributions are associated with the horseshoe structure that would be expected from the wake of an obstacle such as the polymers appear in the baseline condition. These are seen near the edges of the polymer, with the approximate polymer location shown with dashed lines. However, for actuation at $5 \mathrm{~mm}$ downstream, an increase in velocity near $y / D_{h}$ of zero and $z / D_{h}$ of -0.2 is observed, and a decrease at the other edge of the polymer outline is observed at $z / D_{h}$ of 0.2 at the same normalized $y$ coordinate. As mentioned for the single orthogonal configuration, such a decrease at $z / D_{h}$ of 0.2 is not expected, as the polymer should deflect uniformly and an increase on both sides of the polymer would be expected with at least partial symmetry. This increase would be expected, even though the polymer is deflecting in the out of plane direction because the polymer will both push fluid out of plane, but also displace fluid away from its surface in-plane. The local increase in velocity magnitude at $y / D_{h}$ of zero and $z / D_{h}$ of -0.2 occurs for both the 5 and $10 \mathrm{~mm}$ cases. However, for the $10 \mathrm{~mm}$ case, lower velocity magnitudes overall appear in 
the flow. Such a difference in velocities is expected since the polymer's influence should be qualitatively less as velocity is measured further away from the polymer.



Actuation

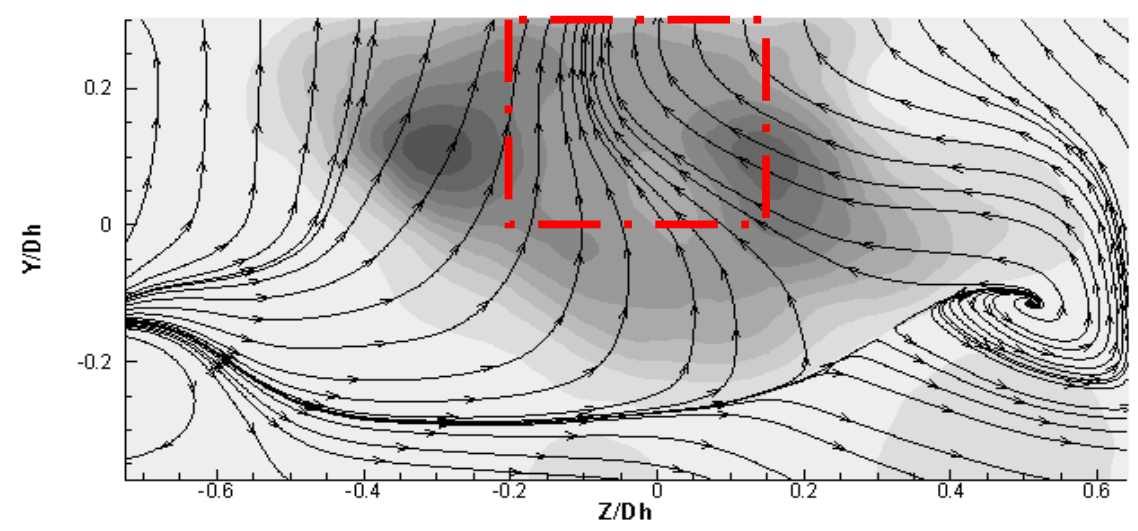

Figure 2.19. Normalized velocity magnitudes for four orthogonally-oriented polymers, $5 \mathrm{~mm}$ downstream. 

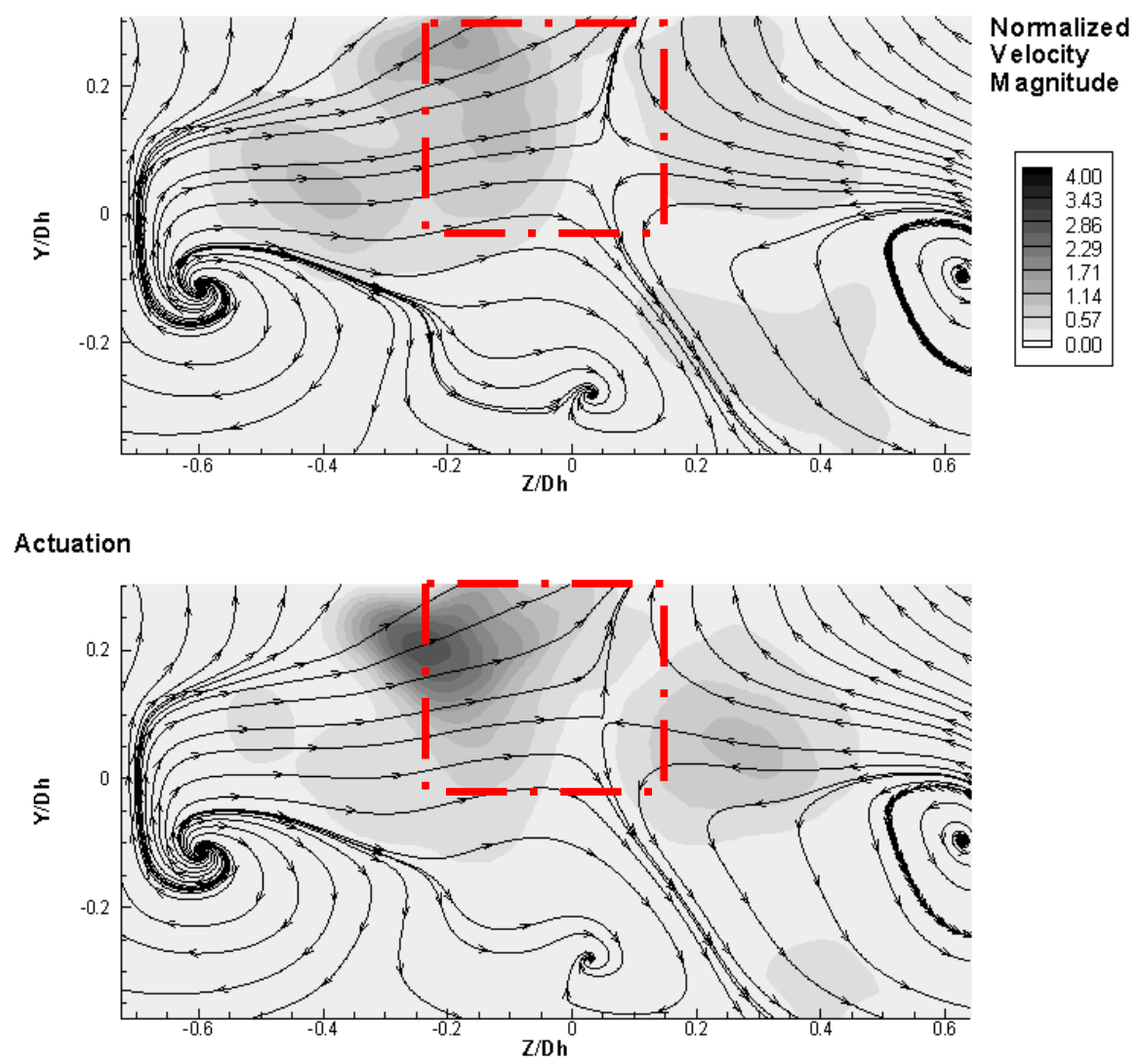

Figure 2.20. Normalized velocity magnitude for four orthogonal polymers, $10 \mathrm{~mm}$ downstream of the last polymer in the configuration.

Examining the percent difference in velocity plot in Figure 2.21, some nonobvious features appear that were not readily apparent from an examination of the timeaveraged contour plots. For the $5 \mathrm{~mm}$ case, an increase commensurate with what was observed in the contour plots of a maximum of $55 \%$ occurs at $y / D_{h}$ of 0.1 and $z / D_{h}$ of -0.3. Also, the velocity deficit at $y / D_{h}$ of 0.1 and $z / D_{h}$ of 0.3 of $-55 \%$ occurs. For the 10 $\mathrm{mm}$ case, many regions where velocity increases of over 200 percent appear, which was not obvious in the velocity contour plots. However, since the downstream velocities are smaller overall for the $10 \mathrm{~mm}$ location compared to the $5 \mathrm{~mm}$ location, subtle differences between the baseline and actuation cases may have been amplified. 

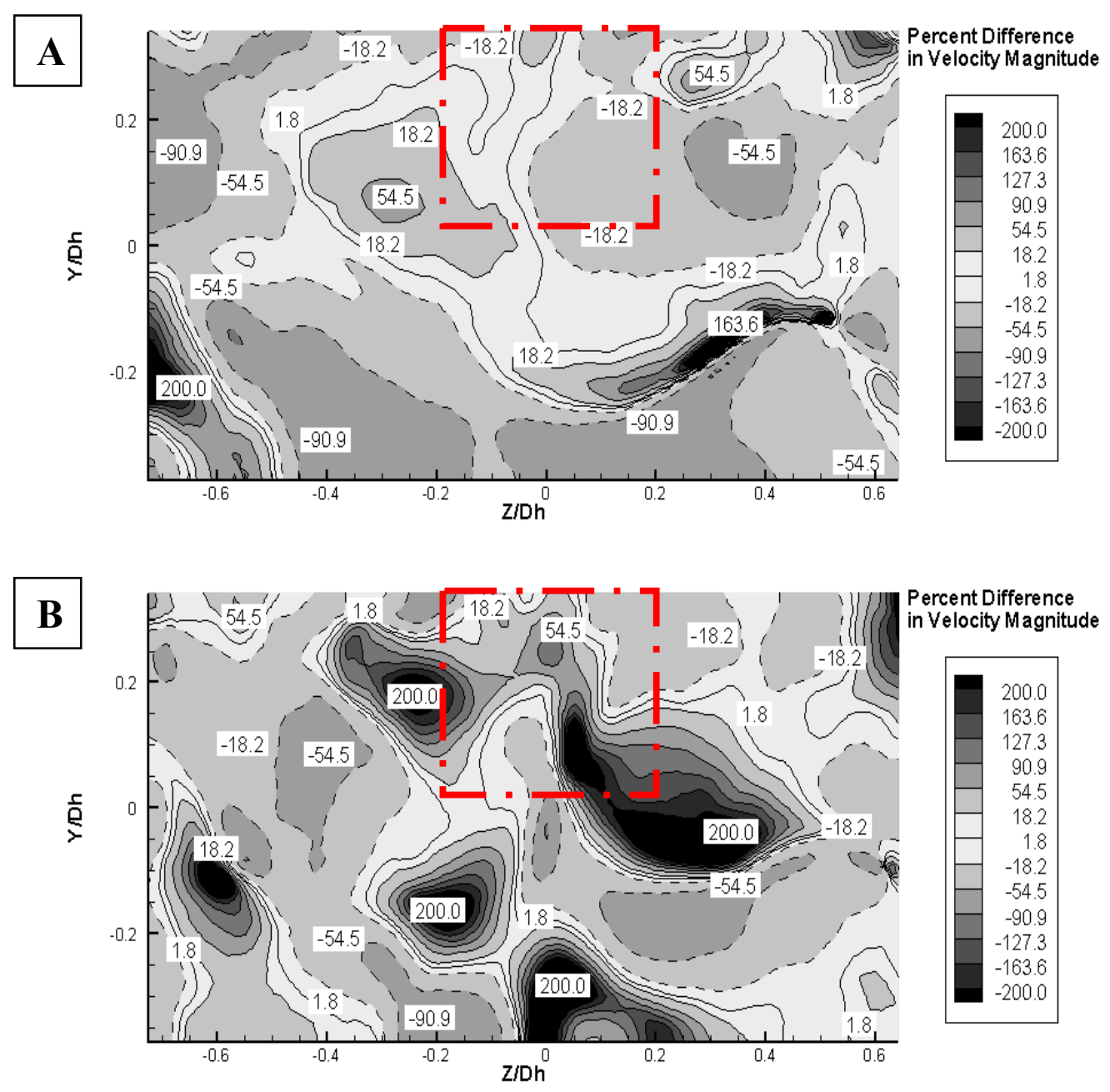

Figure 2.21. Percent difference in velocity magnitude between baseline and actuation cases for 4 orthogonally-oriented polymers at locations of A) 5 millimeters and B) 10 millimeters downstream.

An increase in mixing potential corresponding to the velocity increases noted for the four orthogonal polymer configuration are shown in Figure 2.22 and Figure 2.23. Also, the percent differences for these cases are shown in Figure 2.24. Qualitatively, we also observe that a larger region of high $\xi$ occurs in the data obtained $5 \mathrm{~mm}$ away from the polymer instead of $10 \mathrm{~mm}$, for both baseline and actuation cases. This is as would be expected, since the polymer should exert a greater influence near its surface. For the 5 mm case, amplification in stretching of nearly $150 \%$ occurs for actuation around $y / D_{h}$ of 0.1 and $z / D_{h}$ of -0.3 , correlating with the increase in velocity for the actuation case. Also, 
the reduction in velocity at $y / D_{h}$ of 0.1 and $z / D_{h}$ of 0.3 manifests as a reduction in $\xi$ of nearly $40 \%$.

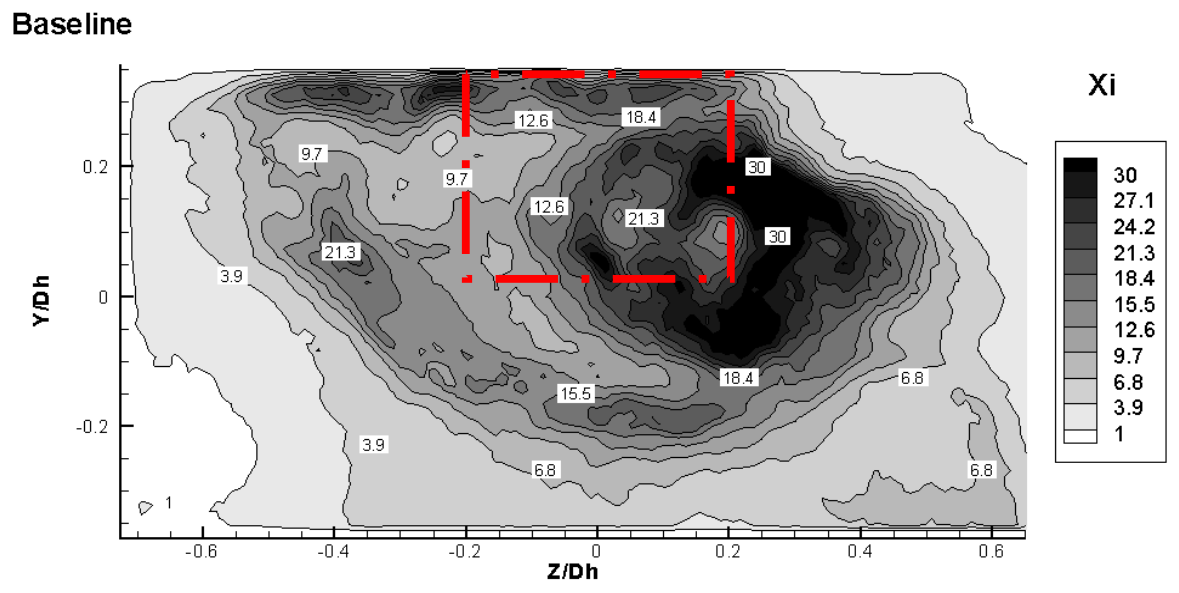

Actuation

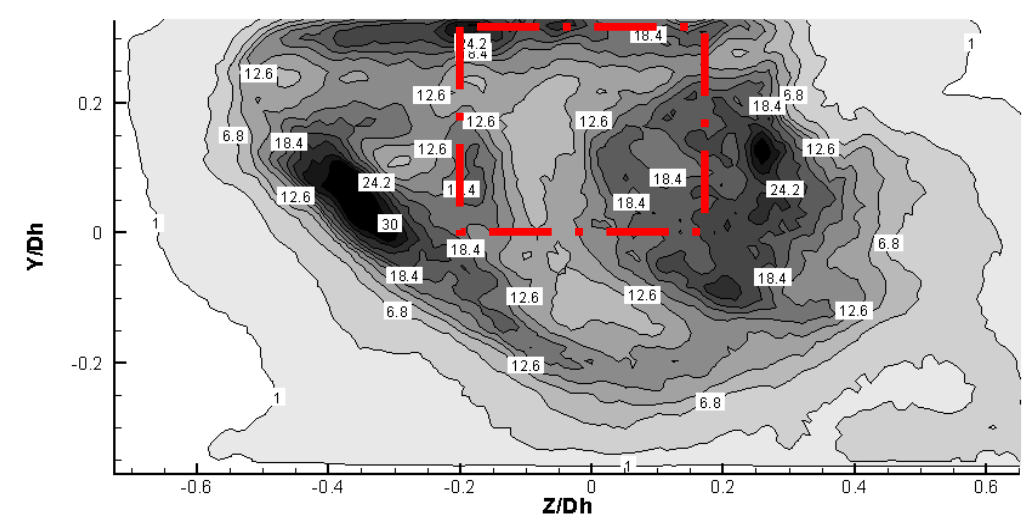

Figure 2.22. Mixing potential of 4 orthogonal polymers at $5 \mathrm{~mm}$ downstream of the last polymer.

Examining the percent difference in $\xi$ for the $10 \mathrm{~mm}$ case, a semi-symmetric increase occurs on either side of the polymers at $y / D_{h}$ of 0.1 and $z / D_{h}$ of -0.2 and $y / D_{h}$ of 0.1 and $z / D_{h}$ of 0.3 . However, the size and shape of the region over which the increase is observed is not similar, even though both of these regions indicate localized increases of nearly $150 \%$. 


\section{Baseline}

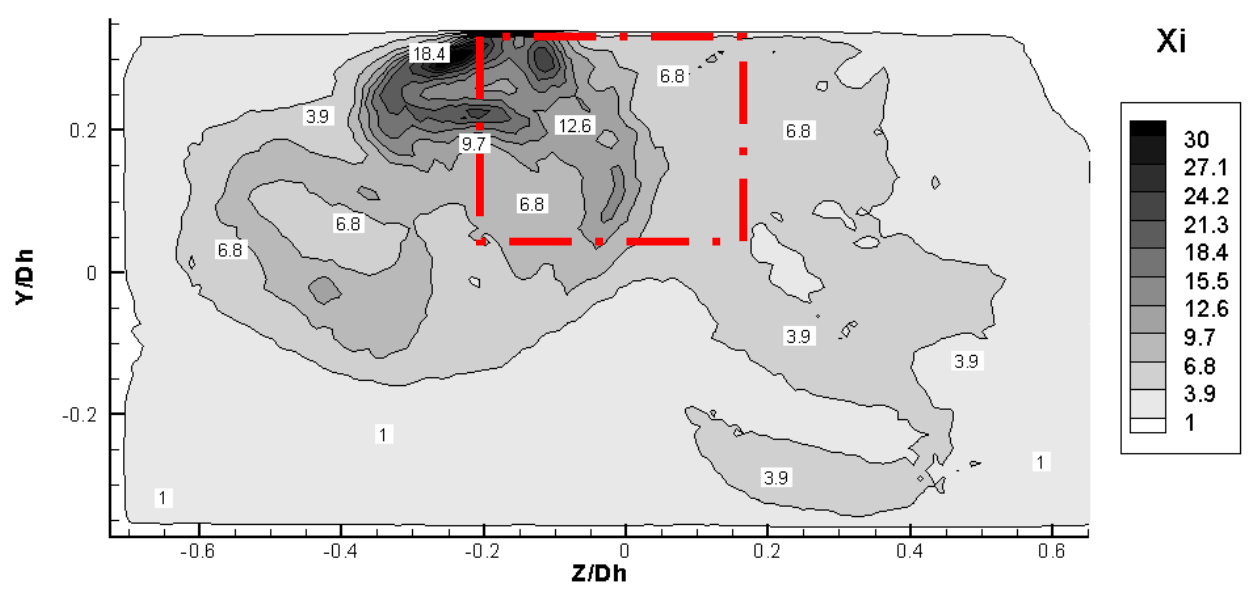

\section{Actuation}

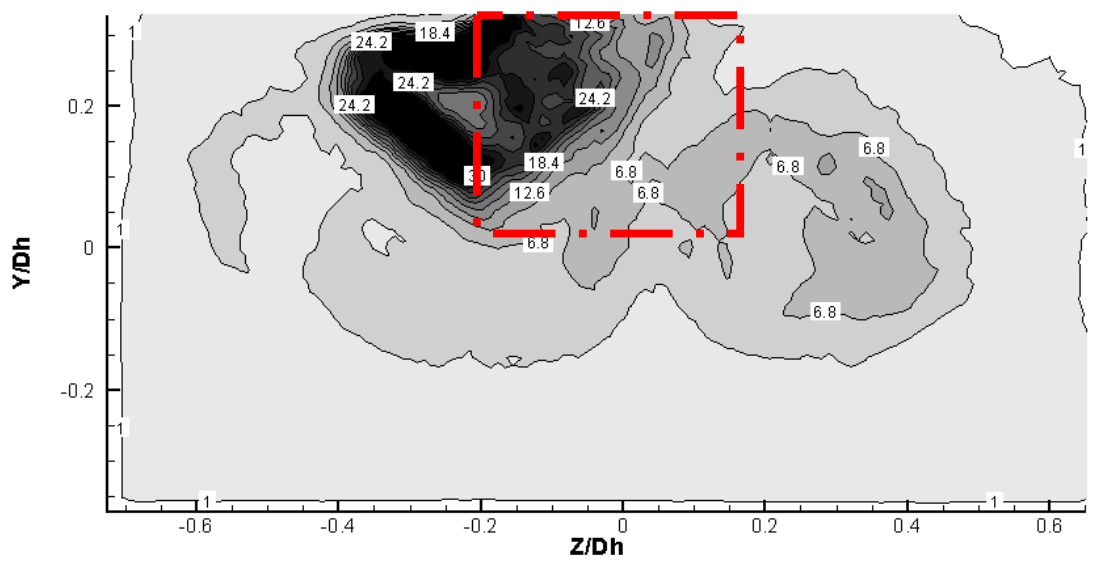

Figure 2.23. Mixing potential for a 4 orthogonal polymer configuration at $10 \mathrm{~mm}$ downstream. 

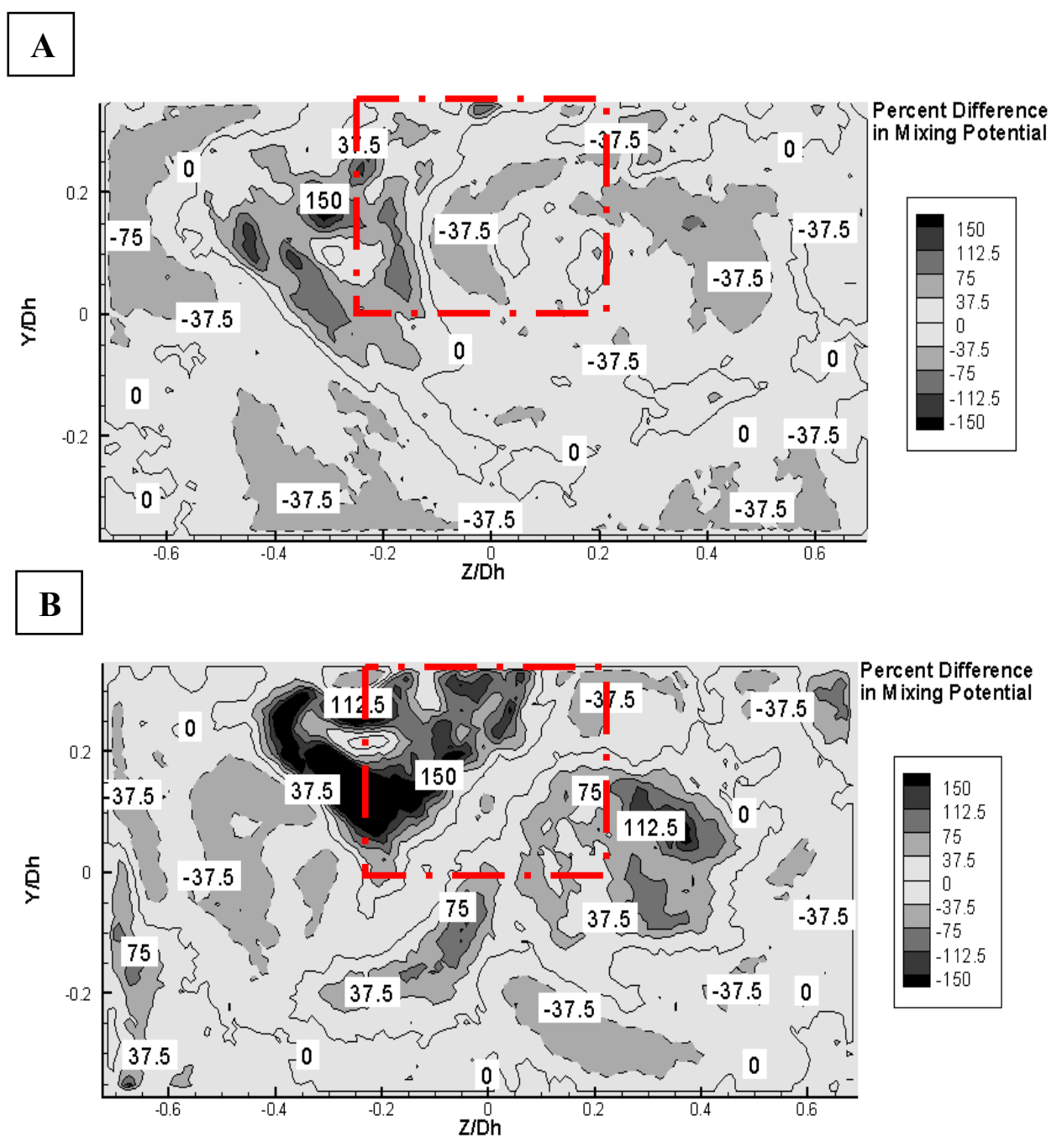

Figure 2.24. Percent difference in mixing potential, $\xi$, for four orthogonal polymers at A) $5 \mathrm{~mm}$ downstream, and B) $10 \mathrm{~mm}$ downstream.

\subsubsection{Time-Resolved Data}

Examining the time-resolved velocity data from the processing provides an additional intuitive insight into the time-averaged data presented previously. This section focuses briefly on the derived flow variable, $\xi$, and results will be shown for one case as an example. For the four orthogonally-oriented polymers, time series plots are shown in 
Figure 2.25 and Figure 2.26 and are calculated by obtaining the average of the mixing potential over the entire field of view for each instant. The results in Figure 2.25 are for the $5 \mathrm{~mm}$ downstream plane. Referring back to previous results for the $5 \mathrm{~mm}$ case, an overall drop in the total time-averaged mixing potential was obtained. This drop is confirmed in the time-resolved data, wherein we see that the magnitude of the strain rate tensor is nearly consistently higher for the baseline case compared to the actuation case. From this data, the mixing potential is relatively constant over time as well for both baseline and actuation cases, and no transient behavior is observed.

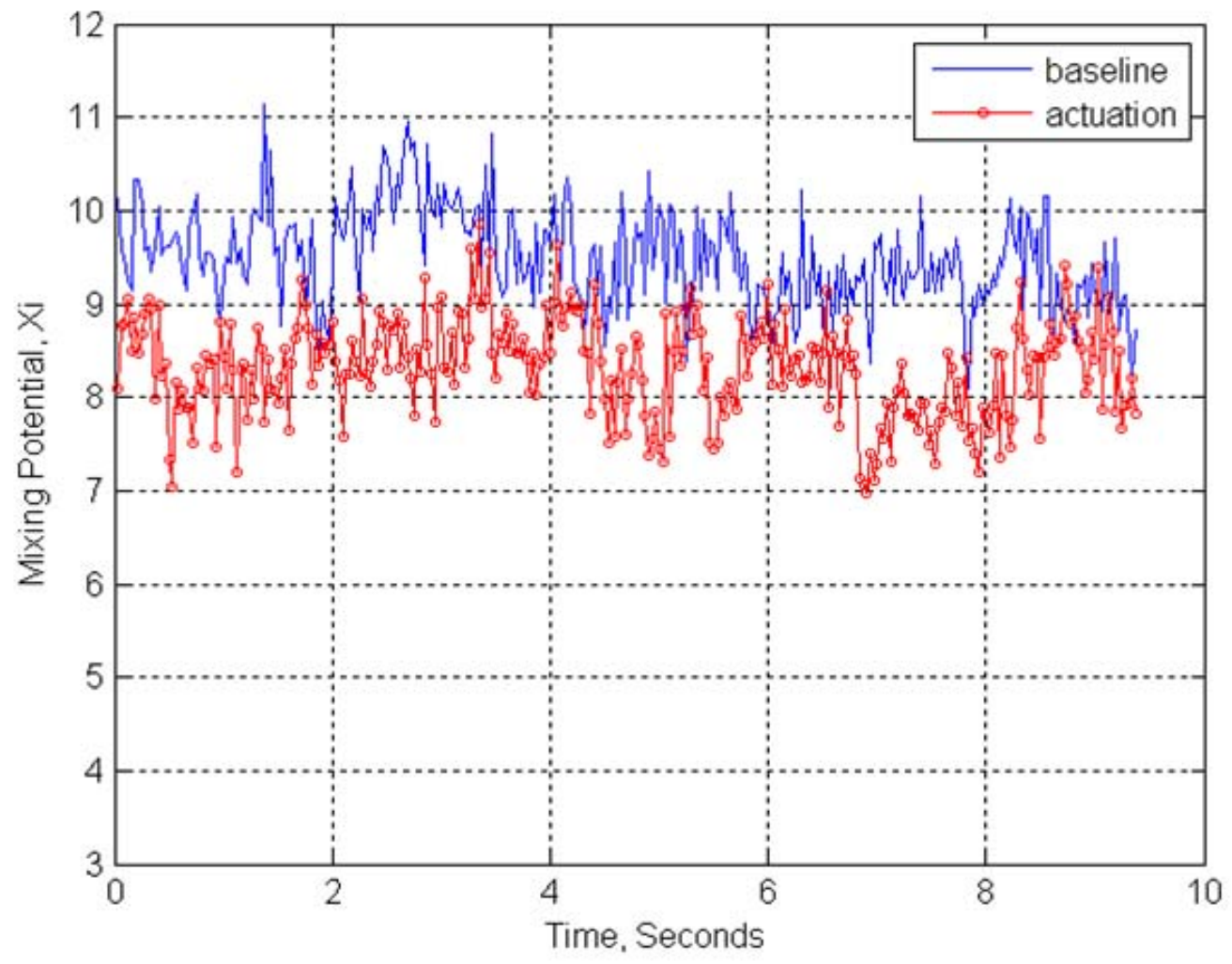

Figure 2.25. Time series of $\xi$ for a 4 orthogonally-oriented polymer case averaged over the field of view at a location $10 \mathrm{~mm}$ downstream of the last polymer in the configuration.

In Figure 2.26, the same information for the $10 \mathrm{~mm}$ downstream plane is plotted. In this case, unlike the $5 \mathrm{~mm}$ plane, a qualitative increase in the magnitude of the strain rate tensor was observed from the time-averaged plots. The amplification also appears in 
the time resolved data shown here, wherein the actuation condition produces nearly consistently higher mixing potential compared to baseline.

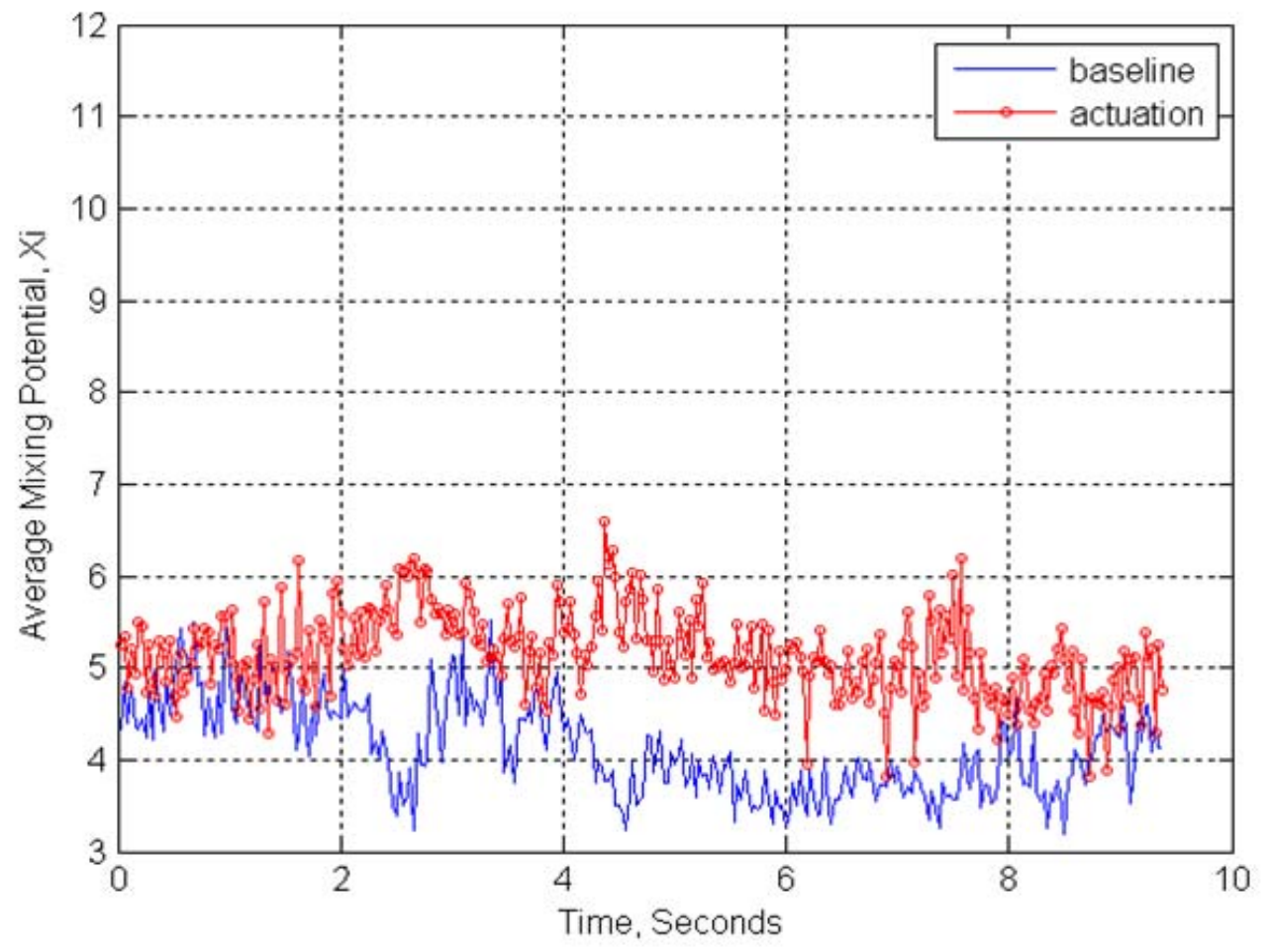

Figure 2.26. Comparison of time-resolved $\xi$ averaged over each instantaneous field of view for a four orthogonal polymer configuration

\subsection{Discussion and Summary of Results}

The percent change in $\xi$ is presented in Figure 2.27 for the entire set of configurations tested in this experiment. The $\xi$ values were calculated by both time and space averaging to arrive at a final value for each time instant in the data, and the reference for the percent difference is the baseline case with cantilevered polymers at rest in the channel. Initially, this data appears to be counter intuitive, in that for each case, the expected trend of higher mixing near the polymer does not appear. 


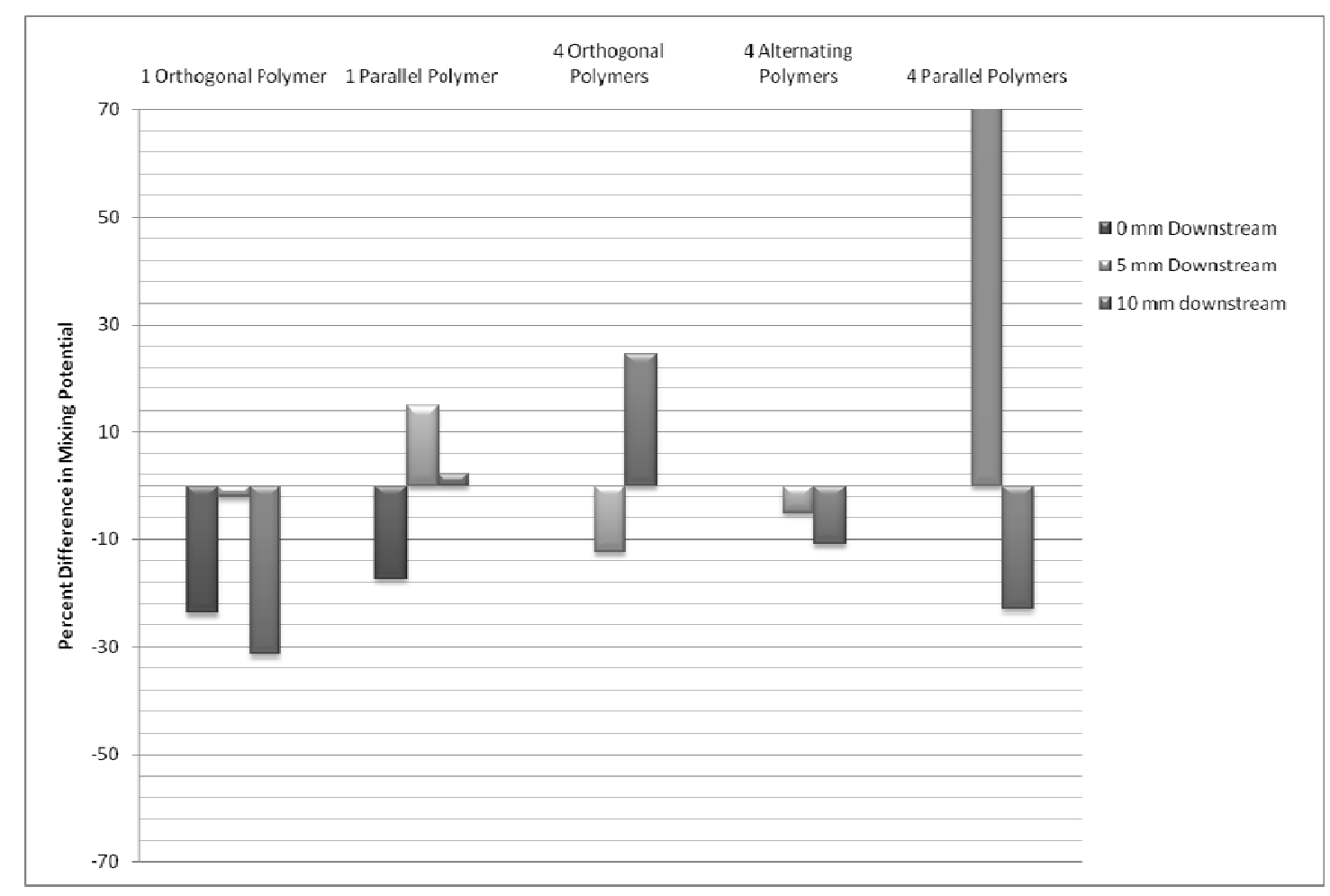

Figure 2.27. Comparison of percent difference of mixing for all configurations tested.

Interestingly, the effect of these polymer cantilevers in the channel becomes much more obvious when the polymer actuation case is compared to the mixing potential in a smooth-walled channel with no polymers present. These data were taken at a plane corresponding to the $5 \mathrm{~mm}$ downstream plane for all cases, and therefore all the comparisons drawn from this data are also made at the $5 \mathrm{~mm}$ downstream case. Again, the percent difference was developed based on averaging both over time and space to arrive at a final value for $\xi$ for both the clean channel as well as the actuation case. The results for all configurations tested in this experiment are shown in Figure 2.28. Based on these results, all cases create a significant increase in mixing potential, with orthogonal-oriented polymers overall performing better than parallel-oriented polymers. Against intuition, the single polymer cases perform as well or better than the multiple polymer cases for parallel and orthogonally-oriented polymers, respectively. For the orthogonal oriented polymers, the single polymer case results in approximately $45 \%$ increase in $\xi$ over the four polymer case. One reason for this result may be that the single polymer used in the one polymer configuration was creating higher strains, and even 
though it was also used in the four polymer cantilever configuration, its impact could have been masked by the other polymers which were not straining as much. Ideally, all the polymers should strain approximately the same amount if they are all driven with the same waveforms, but if the electrodes on some became cracked during the test, or if some of them became dehydrated, the polymer performance can vary widely. However, from the plot in Figure 2.28, we note that the overall impact on the mixing potential of these polymers is a significant one.

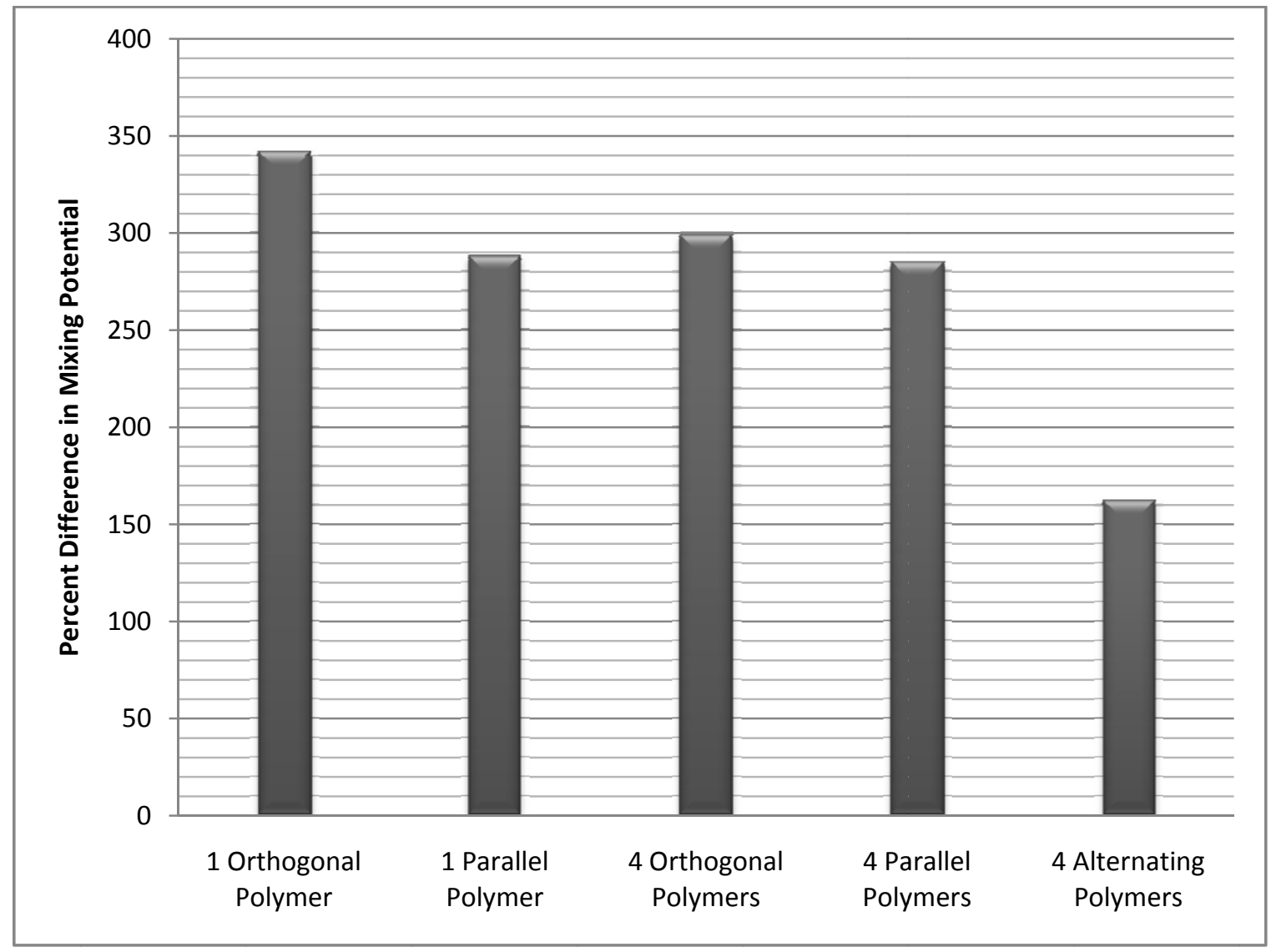

Figure 2.28. Percent difference in $\xi$ based on a clean channel as a baseline, compared to polymer actuation.

In Strook's results, a 90\% increase in mixing was obtained for a Peclet number of $2 * 10^{3}$ over a channel that was $\sim 65$ channel heights long. For our work, the mixing potential has increased by $300 \%$ (for the four polymer case) over 6 channel heights. Since the mixing was evaluated differently, a direct comparison cannot be drawn between 
these two cases, except to note that over fewer channel heights, the presence of these polymers makes a large difference in the flow. Intuitively, we'd expect that the polymers would make more difference over less distance because they are more of an obstruction in the flow than the grooves of Strook's staggered herringbone mixer, which were only 10 percent of the channel height.

Overall, from a comparison of Figures 2.27 and 2.28, it becomes clear that the impact of the polymer is masked by normalizing with respect to the polymer at rest. Particularly since the polymer is a compliant membrane, it is possible that it could experience flow induced vibrations. Therefore, even using these polymers as passive bluff bodies in low Reynolds numbers will induce a low frequency fluttering motion from the compliant polymer membrane that acts to speed mixing. In addition the syringe pump that was used pushes fluid based on the motion of a stepper motor which in turn also induces a small yet measurable disturbance.

Finally, even though a three-dimensional measurement was lacking from this data, it is clear that using cantilevered polymers significantly impacts a highly laminar flow, particularly as seen in Figure 2.28. However, much of the total information of the flow is lost from using planar DPIV to characterize this flow. This is particularly true when calculating $\xi$ to deduce the mixing potential, as the gradients in the streamwise direction cannot be obtained from the data. Therefore, the data presented comparing baseline and actuation cases does not lend itself to immediately drawing conclusions about the complete abilities of ionic polymers in low Reynolds number flows. However, from the plot wherein the polymer actuation is compared to a clean channel, a significant improvement in mixing potential appears due to their presence.

\subsection{Conclusions}

In this work, the potential of Nafion ionic polymer cantilevers was investigated as a means to influence a channel flow with a low Reynolds number and a high Peclet number. Both one and four polymer configurations were tested in which the polymers were aligned either perpendicular or parallel to the streamwise incident flow. Planes of velocity data spanning the channel cross-section were obtained using DPIV. An analysis 
of these velocities shows that polymer actuation can create significant velocity increases in the near-polymer regions. Moreover, using the magnitude of the rate of strain tensor as a quantitative mixing metric, increases are seen in regions near the polymer in which corresponding increases in velocity are also obtained due to polymer actuation. Based on the results presented herein, using Nafion ionic polymer cantilevers as mixing devices for laminar flows appears to have promise. However, results that appear to be inconclusive have been obtained in this experiment. Several possibilities exist as to why the results in this experiment do not agree with our physical intuition, such as a periodicity in the flow structures that would cause regions of large $\xi$ followed by regions in which the flow structures do not appear and the flow is essentially "dead." Another reason could be due to the polymer straining non-uniformly or due to imperfect orientations, as ionic polymers are flexible membranes. Finally, the DPIV data provides a limited picture of the overall flow, and relevant information may have been lost by using this technique to measure the flow. Additional investigations are necessary to fully grasp the physics of using such cantilevers as mixing devices. In this way, the potential of ionic polymer cantilevers to mix laminar flows may be fully exploited to create an effective device. 


\subsection{References}

1. Chiem, N., C. Colyer, and D.J. Harrison. Microfluidic systems for clinical diagnostics, 1997.

2. Chien, R. and J.W. Parce, Multiport Flow-Control System for Lab-on-aChip Microfluidic Devices. Journal of Analytical Chemistry, 2001. 371: p. 106-111.

3. Stremler, M.A., et al., Improving DNA Microarray Hybridization with a Pulsed Source-Sink Mixing Device. Proceedings of the Conference on Microtechnologies in Medicine and Biology, 2005: p. 10-12.

4. Weigl, B.H., R.L. Bardell, and C.R. Cabrera, Lab-on-a-chip for drug development. Advanced Drug Delivery Reviews, 2003. 55(3): p. 349-377.

5. Mitchell, P., Microfluidics -Downsizing Large-Scale Biology. Nature, 2001. 19: p. 717-721.

6. Liu, R.H., et al., Passive mixing in a three-dimensional serpentine microchannel. Journal of Microelectromechanical Systems, 2000. 9(2): p. 190-197.

7. Iacovides, H. and M. Raisee, Recent progress in the computation of flow and heat transfer in internal cooling passages of turbine blades. International Journal of Heat and Fluid Flow, 1999. 20(3): p. 320-328.

8. Buchlin, J.-M., Convective heat transfer in a channel with perforated ribs. International Journal of Thermal Sciences, 2002. 41: p. 332-340.

9. Han, J.C. and Y.M. Zhang, High performance heat transfer ducts with parallel broken and $V$-shaped broken ribs. International Journal of Heat and Mass Transfer, 1992. 35(2): p. 513-523.

10. Stroock, A.D., et al., Chaotic mixer for microchannels. Science, 2002. 295(5555): p. 647-651.

11. Glasgow, I., Aubry, N., Enhancement of Microfluidic Mixing Using Time Pulsing. Lab of A Chip, 2003.

12. Goullet, A., Glasgow, I., Aubry, N., Effects of microchannel geometry on pulsed flow mixing. Mechanics Research Communications, 2006. 33: p. 739-746.

13. Chou, H., M.A. Unger, and S.R. Quake, A Microfabricated Rotary Pump. Biomedical Microdevices, 2001. 3(4): p. 323-330.

14. Selverov, K.P. and H.A. Stone, Peristaltically driven channel flows with applications toward micromixing. Physics of Fluids, 2001. 13(7): p. 1837-1859.

15. Yi, M.Q., H.H. Bau, and H. Hu, Peristaltically induced motion in a closed cavity with two vibrating walls. Physics of Fluids, 2002. 14(1): p. 184-197. 
16. Yang, Z., et al., Ultrasonic micromixer for microfluidic systems. Sensors and Actuators a-Physical, 2001. 93(3): p. 266-272.

17. Moctar, A.O., Aubry, N., and Batton, J., Electro-hydrodynamic microfluidic mixer. Lab on A Chip, 2003. 3: p. 273-280.

18. Glasgow, I., Batton, J., and Aubry, N., Electroosmotic mixing in microchannels. Lab on A Chip, 2004. 4: p. 558-562.

19. Bau, H.H., J. Zhong, and M. Yi, A minute magneto hydro dynamic (MHD) mixer. Sensors and Actuators B: Chemical, 2001. 79(2-3): p. 207215.

20. Kim, Y.-H., Werely, S.T., and Chun, C-H., Phase-resolved Flow Field Produced by a Vibrating Cantilever Plate Between Two Endplates Physics of Fluids, 2004. 16(1): p. 145-162.

21. Shahinpoor, M., et al., Ionic Polymer-Metal Composites (IPMCs) as Biomimetic Sensors, Actuators, and Artificial Muscles. Journal of Smart Materials and Structures 1998. 7: p. R15-R30.

22. Akle, B.J., M.D. Bennett, and D.J. Leo, Ionic Electroactive Hybrid Transducers. Proceedings of SPIE 2005. 5759: p. 153-164.

23. Akle, B.J., Multilayer Ionic Transducers, in Mechanical Engineering. 2003, Virginia Polytechnic Institute and State University Blacksburg. p. 102.

24. White, F.M., Viscous Fluid Flow (Second ed). McGraw-Hill, Inc. New York, 1991.

25. Adrian, R.J., Dynamic ranges of velocity and spatial resolution of particle image velocimetry. Measurement Science \& Technology, 1997. 8: p. 1393-1398.

26. Adrian, R.J., Twenty years of particle image velocimetry. Experiments in Fluids, 2005. 39(2): p. 159-169.

27. Westerweel, J., Fundamentals of digital particle image velocimetry. Measurement Science \& Technology, 1997. 8(12): p. 1379-1392.

28. Huang, H., D. Dabiri, and M. Gharib, On errors of digital particle image velocimetry. Measurement Science \& Technology, 1997. 8(12): p. 14271440.

29. Zalc, J.M., Szalai, E.S., Muzzio, F.J., Jaffer,S., Characterization of Flow and Mixing in an SMX Static Mixer. AICHE Journal 2002. 48(3): p. 427436.

30. Hobbs, D.M., P.D. Swanson, and F.J. Muzzio, Numerical characterization of low Reynolds number flow in the Kenics static mixer. Chemical Engineering Science, 1998. 53(8): p. 1565-1584. 


\section{Chapter 3: Conclusions and Recommendations}

\subsection{Discussion and Conclusions}

This thesis presents a first experiment to use Nafion ionic polymers in a cantilevered configuration as active mixing mechanisms in a low Reynolds number channel flow. Single-pulsed DPIV data was acquired and captured the cross-section of the flow at several locations downstream of the cantilevered polymers, which were approximately half of the channel height and one third of the channel width. Both single polymers as well as four polymer configurations were tested in the experiment in an attempt to evaluate the mixing capabilities of different orientations and numbers of polymers. Since the polymers at rest create secondary flows from obstructions, both baseline and actuation cases were captured to determine the difference, which represents the polymer's impact on the flow.

Post-processing revealed subtle differences when comparing baseline and actuation cases, although when calculating the percent difference in velocity magnitude, significant values appear. This indicates that the polymer has an effect on the flow, albeit an unclear one. Because the purpose of these polymers is to perform mixing in a laminar flow, a quantitative means was employed to describe the local stretching based on the velocity fields obtained using DPIV. A mixing metric defined by other groups based on the magnitude of the two-dimensional strain rate tensor was used to evaluate how the polymer changed the mixing potential of the flow. The magnitude of the rate of strain tensor represents how many interfaces are being created in the flow, which in turn speeds diffusion. For some cases, the polymer cantilevers created significant increases in mixing potential. However, this change in mixing potential cannot be readily correlated across all configurations tested in this experiment. Moreover, the rate of strain tensor does describe exactly how well the flow is mixed; it merely estimates the potential of mixing of a specific flow field. 
Nafion ionic polymers have significant potential to be used as low Reynolds number mixing devices due to their practicality in a demanding environment, but the results of this work have proven to be inconsistent, in that not all of the cases tested show a readily describable trend. To understand how well the polymer works as a vibrating cantilever, additional work is necessary to obtain more information with respect to quantifying the complete three-dimensional flow field. Some methods that could lead to a more successful experiment are described in the next section.

\subsection{Future Work}

Although the fluid structures of this channel flow were captured with fidelity, the mixing metric used to describe mixing potential of the flow provides only a limited view of the total mixing in the channel. Therefore, a dye-based mixing analysis should be combined with DPIV in future experiments to both determine the mixing characteristics of cantilevered polymers as well as measure the structures in the flow. Dye-based mixing quantification also uses optical measurement, but unlike DPIV, the dye carries the threedimensional impact of geometry or otherwise with it as it convects. Therefore, a better understanding of the total mixing in the channel can be obtained if this technique is used that will not be limited by the two-dimensionality of conventional DPIV.

Several different improvements should be enacted in future experiments to improve the quality of the results. The first improvement involves the polymer type to use to create the largest deflection possible. Direct Assembly Process (DAP) polymers swollen with ionic liquid instead of water can generate much higher deflections in air than the ionic polymers used in this experiment. The disadvantage of using ionic liquids as the imbibed fluids is that they are hydrophilic. Therefore, DAP polymers must be encapsulated before use in water to keep the ionic liquid inside of the Nafion membrane. The encapsulation of polymers currently is typically done using a hot press and Mylar film. An attempt was made to use these polymers in a preliminary test, but very small deflections on the same order as the water-based polymers were obtained. One reason as 
to why this encapsulation method did not work is that the Mylar film is water permeable, and will allow water to diffuse across thus damaging the actuator. Additionally, the encapsulation process, which uses a hot press to seal the Mylar around the polymer, appears to create cracks in the electrode material. This is another possibility as to why small deflections were obtained. A way to circumvent this issue might be to use Kapton for the encapsulation material or to attempt to use thicker Mylar sheet to protect the polymer. The downside to both of these ideas is the increased resulting stiffness of a cantilever, and hence, a possibility for smaller deflections. Yet another means to increase the polymer deflections would be to continue using the water-based polymers, but to stack them in layers to improve their authority. Again here, the stiffness of the final composite would be much greater and may lead to some decrease in performance.

Additionally, some variation in the manufacturing process is present with the polymers used in this experiment, since gold leaf is manually sprayed onto the Nafion membrane to create the electrodes, as mentioned in Chapter 2. This leads to a potential for unrepeatability in the polymer deflections and could partly explain why the polymers did not perform predictably in this work. The improvement of consistency in manufacturing is the subject of research of other members of Vlachos' research group. In addition, ionic polymers are typically useable to actuations for up to 250,000 cycles for which they can produce a predictable deflection based on an applied voltage potential. However, within the first few thousand cycles, there is a transient period during which the polymer response to voltage will be higher. By 10,000 cycles into the polymer's lifespan, the response levels-out and stays virtually the same until the end of its useful number of cycles [1].

Beyond improvements in the polymers, the quality of flow as well as the test section should be reexamined as part of any further experiments. As shown in Appendix $\mathrm{B}$, swirls appear in the data, which is due to pulsatility introduced from the syringe pump used to drive the flow. Instead of a motor-based pump, a gravity-fed channel flow would be preferable to improve flow steadiness, and at least one group has used such a setup successfully in experiments that depend on eliminating pulsatility effects [2]. 
In addition, the test section size compared to the deflection amplitude of the polymers was also not desirable, since the test section area was over $300 \mathrm{~mm}^{2}$ while the polymer deflected approximately $2 \mathrm{~mm}$ peak to peak. Therefore, whole channel mixing structures readily attributable because of cantilevered polymer deflections would be unlikely, particularly over such short distance for mixing. One way to increase the impact of the polymers used in this experiment would be to decrease the cross-sectional area of the test section. Also, very little actuation length was used in comparison to other mixing devices for laminar flows. Comparing to Strook's [3] work, a channel that is relatively $1 / 8$ as long was used in this work, and only part of the channel contained polymers. An improvement in whole-field mixing potential may be obtained by using more actuators over a longer length.

In summary, many changes may be undertaken to obtain improved data that clearly documents the effectiveness and potential of ionic polymer transducers for laminar mixing. Repeating the experiment using a similar setup also has merit, but additional ensembles of data should be acquired to develop repeatability in the measurements. If the effect of polymer actuation can be amplified as well as some additional improved studies to determine exactly the wake and flow structures created by polymer actuation, the concept of using ionic polymers as a mixing device can be further matured. If cantilevered polymers can be used as an effective, efficient laminar mixer, the development and prevalence of microfluidics in technology could sustain significant growth

\subsection{References}

1. Bennett, M.D., and Leo, D.J., Manufacture and characterization of ionic polymer transducers employing non-precious metal electrodes. Smart Materials and Structures, 2003. 12: p. 424-436.

2. Sharp, K.V., and Adrian, A.D., Transition from laminar to turbulent flow in liquid filled tubes. Experiments in Fluids, 2004. 36: p. 741-747.

3. Stroock, A.D., et al., Chaotic mixer for microchannels. Science, 2002. 295: p. 647-651. 


\section{Vita}

Alicia Williams grew up in Fairfax, Virginia and graduated from Fairfax High School in 2001. She proceeded to her undergraduate education at Virginia Tech in mechanical engineering and received her bachelor's degree in 2005. Also in 2005, she was selected to receive a National Science Foundation Graduate Research Fellowship to fund her graduate studies, which she has pursued at Virginia Tech. She plans to continue her education at Virginia Tech for her doctorate in mechanical engineering. 
